Fátima das DoRes da CRUZ

\title{
Avaliação dos componentes da qualidade de vida na clínica de insuficiência
}

\section{cardíaca}

Tese apresentada à Faculdade de Medicina da Universidade de São Paulo para obtenção do título de Doutor em Ciências.

Área de concentração: Cardiologia

Orientador: Prof. Dr. Edimar Alcides Bocchi

São Paulo 
Dados Internacionais de Catalogação na Publicação (CIP)

Preparada pela Biblioteca da

Faculdade de Medicina da Universidade de São Paulo

Creprodução autorizada pelo autor

\section{Cruz, Fátima das Dores da}

Avaliação dos componentes da qualidade de vida na clínica de insuficiência cardíaca / Fátima das Dores da Cruz. -- São Paulo, 2010.

Tese(doutorado)--Faculdade de Medicina da Universidade de São Paulo. Departamento de Cardio-Pneumologia.

Área de concentração: Cardiologia.

Orientador: Edimar Alcides Bocchi.

Descritores: 1.Qualidade de vida 2.Insuficiência cardíaca 3.Educação de pacientes como assunto

USP/FM/SBD-084/10 
"Não acrescente dias a sua vida, mas vida aos seus dias".

(Harry Benjamin) 


\section{Dedicatória}


Á Deus por estar sempre em meu caminho me direcionando e fortalecendo em todos os momentos, especialmente nos mais difíceis com o seu amor e sua paz.... 
A meu pai José Cardoso da Cruz (in memoriam), pelo exemplo de vida, trabalho, honestidade, integridade e caráter. Por ter me guiado e ensinado a importância do estudo, trabalho, respeito e honestidade e acima de tudo, do amor e dos grandes valores da vida. Por estar sempre ao meu lado e pela sua presença e apoio em todos os momentos, especialmente nos mais difíceis, e a quem eu agradeço tudo o que sou hoje.

À minha querida mãe Maria dos Prazeres da Cruz, que sempre esteve e está ao meu lado em todos os momentos, por caminhar sempre junto comigo para que eu nunca desista, pelo seu grande e incondicional amor e carinho. 
Agradecimentos 


\section{Agradecimento Especial}

Ao Professor Dr. Edimar Alcides Bocchi, pela oportunidade de me confiar à coordenação da Clínica de Insuficiência Cardíaca e o desenvolvimento deste trabalho, pela paciência, dedicação e competência com a formação e o rigor científico, e especialmente por ser meu orientador.

A minha incessante gratidão aos pacientes da clinica de insuficiência cardíaca, parceiros fundamentais na realização deste estudo.

A todos que de uma forma ou outra me apoiaram nesta caminhada,

Muito Obrigada! 


\section{Agradecimentos}

Ao Dr. Victor Sarli Issa, Dr. José Ramon Lanz e Dra Silvia Ayub Ferreira, pelo grande auxilio e apoio na realização deste estudo.

Ao Prof. Dr. Luiz Felipe Pinho pela grande colaboração na elaboração da análise estatística deste estudo.

Ao Dr. Paulo Roberto Chizzola e Dr. Germano Emilio Conceição pelo apoio e colaboração.

Ao Prof. Dr. Fernando Bacal e Dr. Philipe Vieira Pires por me incentivarem a realizar este estudo e especialmente por estarem sempre à disposição em me ajudar.

A toda equipe médica, assistentes, residentes e estagiários pelo apoio e dedicação aos pacientes da Clínica de Insuficiência Cardíaca.

Á equipe interdisciplinar da Clínica de Insuficiência Cardíaca pelo profissionalismo e excelente trabalho, colaboração e dedicação no atendimento aos pacientes.

Aos bolsistas e estagiários de enfermagem pela ajuda na realização do teste de qualidade de vida e monitorização dos pacientes e pelo excelente trabalho e dedicação.

Á secretaria de pós graduação pela dedicada colaboração.

A todos os amigos e profissionais da Unidade de Insuficiência Cardíaca que me auxiliaram para o desenvolvimento deste trabalho. 
Normatização Adotada 
A elaboração desta tese está de acordo com as seguintes normas, em vigor no momento da publicação:

Referências: adaptado do International Commitee of Medical Journals Editors (Vancouver)

Universidade de São Paulo. Faculdade de Medicina. Serviço e Documentação. Guia de apresentação de dissertações, teses e monografias. Elaborado por Annelise Carneiro da Cunha, Maria Julia de A. L. Freddi, Maria Fazanelli Crestana, Marinalva de Souza Aragão, Suely Campos Cardoso, Valéria Vilhena. $2^{a}$ ed. - São Paulo: Serviço de Biblioteca e Documentação - SBD/ FMUSP, 2005.

Abreviaturas dos títulos dos periódicos de acordo com List of Journals Indexed in Index Medicus. 
Sumário 


\section{SUMÁRIO}

Lista de Abreviaturas

Listas de Símbolos

Lista de Tabelas

Lista de Figuras

Resumo

Summary

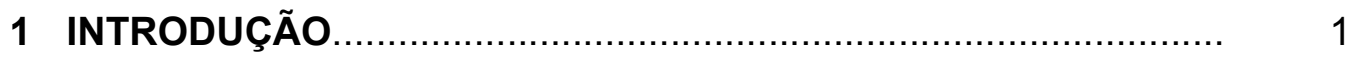

1.1 Importância da insuficiência cardíaca................................. 2

1.2 Complexidade o tratamento da insuficiência cardíaca........... 5

1.3 Importância da educação e monitorização.......................... 7

1.4 Qualidade de vida na insuficiência cardíaca....................... 8

1.5 Hipótese do estudo.............................................. 12

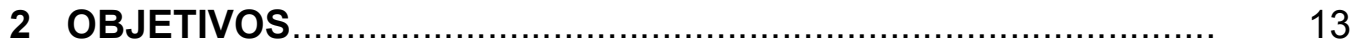

2.1 Objetivos primários........................................................ 14

2.2 Objetivos secundários............................................ 14

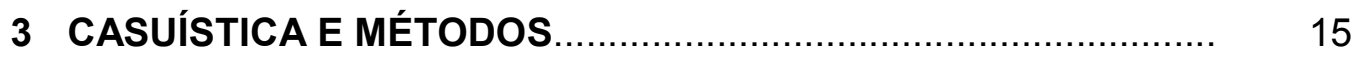

3.1 População.......................................................... 16

3.2 Dinâmica do estudo............................................. 17

3.3 Qualidade de vida.................................................. 24

3.4 Análise estatística................................................. 27

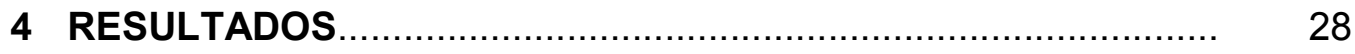


Dados demográficos.

4.1 Qualidade de vida, número de atendimentos em unidades de emergência, número de internações e número de dias de internação.

4.2 Qualidade de vida.......................................................... 32

4.2.1 Qualidade de vida - Resultado global................................. 32

4.2.2 Qualidade de vida - Dimensão física.................................... 33

4.2.3 Qualidade de vida - Dimensão emocional............................. 34

4.2.4 Qualidade de vida - Demais questões................................. 35

4.3 Qualidade de vida - Gêneros................................ 36

4.3.1 Qualidade de vida - Gênero masculino....................... 36

4.3.2 Qualidade de vida - Gênero feminino....................... 37

4.4 Analise univariada.......................................... 38

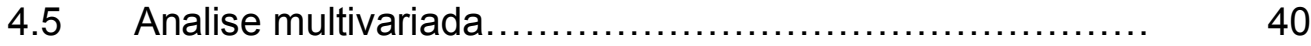

$4.6 \quad$ Sobrevida.................................................... 42

4.6.1 Sobrevida..................................................... 42

4.6.2 Sobrevida livre de eventos.................................... 43

4.6.3 Sobrevida e qualidade de vida basal........................ 44

4.6.4 Sobrevida livre de eventos e qualidade de vida basal......... $\quad 45$

4.6.5 Sobrevida e qualidade de vida no seguimento..................... 46

4.6.6 Sobrevida livre de eventos e qualidade de vida no

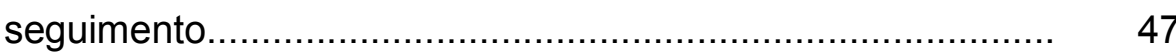

5 DISCUSSÃO

$5.1 \quad$ Implicações Clínicas........................................... 54 
5.2 Limitações do Estudo............................................ 55

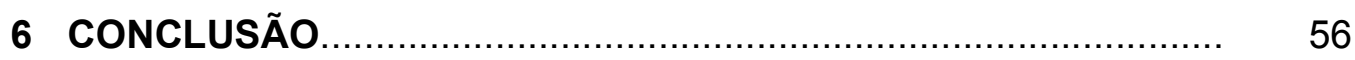

7 ANEXOS

7.1 Minnesota Living with Heart Failure Questionnaire............. 59

8 APENDICE

8.1 Foto dos pacientes eem reunião na clínica de insuficiência

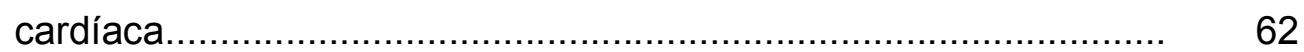

9 REFERÊNCIAS BIBLIOGRÁFICAS ............................................... 63 
Listas 


\section{LISTA DE ABREVIATURAS}

\begin{tabular}{|c|c|}
\hline IC & = Insuficiência cardíaca \\
\hline QV & = Qualidade de vida \\
\hline MLWHFQ & = Minnesota Living with Heart Failure Questionnaire \\
\hline DF & = Dimensão Física \\
\hline DE & = Dimensão Emocional \\
\hline DQ & = Demais Questões \\
\hline $\mathrm{CF}$ & $=$ Classe funcional \\
\hline GI & = Grupo Intervenção \\
\hline GC & $=$ Grupo Controle \\
\hline pts & $=$ pontos \\
\hline DSVE & = Diâmetro sistólico do ventrículo esquerdo \\
\hline DDVE & = Diâmetro diastólico do ventrículo direito \\
\hline FEVE & = Fração de ejeção do ventrículo esquerdo \\
\hline $\mathrm{VO}_{2}$ & = Consumo máximo de oxigênio \\
\hline NYHA & $=$ New York Heart Association \\
\hline OMS & = Organização Mundial de Saúde \\
\hline REMADHE & $=$ Repetitive Education and Monitoring for Adherence in \\
\hline
\end{tabular}




\section{LISTA DE SIMBOLOS}

$\begin{array}{ll}\% & \text { Porcentagem } \\ < & \text { Menor que } \\ > & \text { Maior que } \\ = & \text { Igual a } \\ \pm & \text { Mais ou menos } \\ \text { Kg/min } & \text { Quilograma por minuto }\end{array}$




\section{LISTA DE TABELAS}

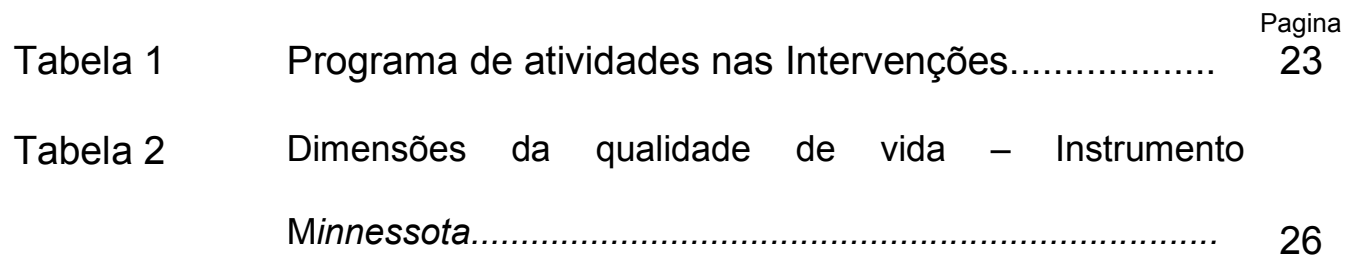

Tabela 3 Características basais dos grupos controle e intervenção...................................................... 30

Tabela 4 Analise dos univariada dos fatores associados a sobrevida................................................. 38

Tabela 5 Analise univariada dos fatores associados a eventos...................................................... 39

Tabela 6 Analise multivariada dos fatores associados a sobrevida.................................................... 40

Tabela 7 Analise multivariada - livre de eventos (óbito e

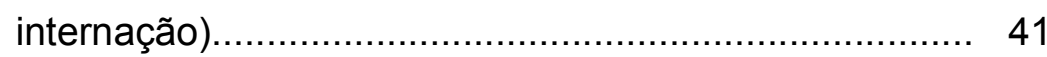




\section{LISTAS DE FIGURAS}

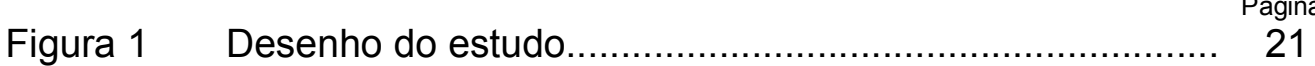

Figura 2 Fluxograma do estudo....................................... 22

Figura 3 Qualidade de vida, número de atendimentos em unidades de emergência, número de internações e número de dias de internação..................................... 31

Figura 4 Qualidade de vida - resultado global........................... 32

Figura 5 Qualidade de vida - dimensão física......................... 33

Figura 6 Qualidade de vida - dimensão emocional.................... 34

Figura $7 \quad$ Qualidade de vida - demais questões........................... 35

Figura 8 Qualidade de vida - resultado global - gênero

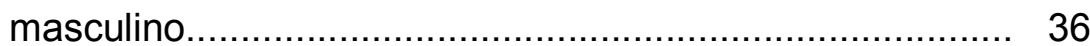

Figura 9 Qualidade de vida - resultado global - gênero

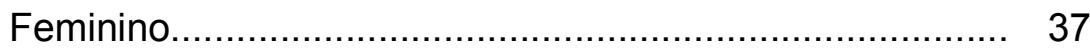

Figura $10 \quad$ Curva de sobrevida................................................. 42

Figura 11 Curva de sobrevida - livre de eventos.......................... 43

Figura 12 Curva de sobrevida e qualidade de vida basal................ 44

Figura 13 Curva de sobrevida livre de eventos e qualidade de vida basal..................................................................... 45

Figura 13 Curva de sobrevida e qualidade de vida no seguimento... 47

Figura 14 Curva de sobrevida livre de eventos e qualidade de vida no seguimento 
Resumo 


\section{RESUMO}

Cruz F. D. Avaliação dos componentes da qualidade de vida na clínica de insuficiência cardíaca. São Paulo, 2010. Tese de Doutorado - Instituto do Coração - Faculdade de Medicina, Universidade de São Paulo.

Fundamento: A insuficiência cardíaca (IC) é uma síndrome, de alta prevalência, comprometendo a qualidade de vida (QV).

Objetivo: Testamos a hipótese de que um programa seqüencial de educação e monitorização (DMP), pode modificar os componentes do questionário Minnesota Living Heart Failure Questionnaire (MLHFQ) aplicado a pacientes com IC em seguimento ambulatorial.

Métodos: Esta investigação é uma extensão do estudo REMADHE, prospectivo, randomizado, com grupo intervenção (GI) submetido a um DMP, versus grupo controle (GC). A QV foi avaliada pelo MLHFQ, aplicado na inclusão no estudo, a cada seis meses até os dois anos de seguimento, $\mathrm{e}$ após anualmente. Incluímos 412 pacientes, $60,5 \%$ do sexo masculino, e fração de ejeção de ventrículo esquerdo de $34,7 \pm 10,5 \%$.

Resultados: No Gl ocorreu melhora significativa em todos os componentes do MLHFQ ( $53 \pm 23 v s .29 \pm 19, p=0,007)$, da dimensão física ( $24 \pm 10$ vs. $13 \pm 9$, $p=0,0002)$, da dimensão emocional $(13 \pm 7 v s .9 \pm 7, p=0,02)$ e demais questões ( $21 \pm 9 \mathrm{vs} .11 \pm 7, p=0,001)$. No Gl houve melhora de sobrevida livre de eventos (óbito e internação) $(p<0,001)$, houve relação entre o escore da $Q V$ após a inclusão e a sobrevida, mas não com a QV de vida basal ( $p=0,7)$. A QV demonstrou ser um fator independente na determinação de sobrevida livre de eventos. $\mathrm{Na}$ avaliação dos gêneros, ambos demonstraram melhora significativa, mas no masculino a melhora ocorreu tardiamente em relação ao feminino.

Conclusão: Este programa de educação e monitorização continua em longo prazo melhorou a QV e seus componentes em pacientes em acompanhamento ambulatorial. Entretanto, os componentes da QV podem responder diferentemente a intervenção.

Descritores: 1. Qualidade de vida, 2. Insuficiência cardíaca, 3. Educação de pacientes. 
Summary 


\section{SUMMARY}

Cruz F. D. Components of Life Quality Evaluation in Heart Failure Clinic. São Paulo, 2010. Tese de Doutorado - Faculdade de Medicina, Universidade de São Paulo.

Background: Heart failure (HF) is a high prevalence syndrome, pledging the quality of life $(\mathrm{QL})$.

Objective: We tested the hypothesis that a sequential program of education and monitoring (DMP) may modify the components of Minnesota Heart Failure Living Questionnaire (MLHFQ) on outpatient patients with HF.

Methods: This research is an extension of the REMADHE study, prospective, randomized with an intervention group (IG) subjected to a DMP, versus a control group (CG). QL was evaluated by MLHFQ applied the inclusion in the study, every six months to the following-up two years, and thereafter annually. We included 412 patients, $60.5 \%$ were male, and $34.7 \pm$ $10.5 \%$ of left ventricle ejection fraction.

Results: In GI, there was a significant improvement in all MLHFQ components (53 $\pm 23 v s .29 \pm 19, p=0.007)$, physical dimension ( $24 \pm 10$ vs. $13 \pm 9, p=0.0002)$, emotional dimension ( $13 \pm 7 \mathrm{vs} .9 \pm 7, p=0.02$ ) and other issues ( $21 \pm 9 v s .11 \pm 7, p=0.001)$. In Gl, there was an improvement in event-free survival (death and hospitalization) $(p<0.001)$ there was a relation between the QL scores after inclusion and survival, but not with the life baseline QL ( $p=0.7)$. Quality of life proved to be an independent factor in determining event-free survival. In genres assessment, both showed significant improvement, but in males the improvement occurred late in relation to females.

Conclusion: This education and monitoring program continues for a long term to improve QL and its components on the follow up of outpatient patients. However, the components of QL may respond differently to intervention.

Keywords: 1. Quality of life, 2. Heart Failure, 3. Education patients. 


\section{Introdução}


A síndrome de insuficiência cardíaca (IC) está associada a prognóstico reservado e a freqüentes morbidades, tendo alta prevalência no Brasil e no mundo. Requer cuidados contínuos e gastos elevados o que ocasiona limitações às atividades diárias, piora da qualidade de vida (QV) e determina a necessidade de mudanças do estilo de vida dos pacientes. Um programa de manejo de doença crônica tem sido proposto, devido à complexidade do manejo da insuficiência cardíaca.

A avaliação da qualidade de vida tem se tornado cada vez mais indispensável no seguimento da prática clínica de pacientes com IC. Tem-se observado uma preocupação da comunidade científica em identificar os determinantes de qualidade de vida, especialmente em situações de doenças crônicas, com o objetivo de desenvolver estratégias de melhora de qualidade de vida.

\subsection{IMPORTÂNCIA DA INSUFICIÊNCIA CARDÍACA.}

A IC é a via final comum da maioria das doenças que acometem o coração, sendo um dos mais importantes desafios clínicos atuais na área da saúde. Trata-se de um problema epidêmico em progressão. ${ }^{1,2}$

A IC é uma síndrome clínica complexa de caráter sistêmico, definida como disfunção cardíaca que ocasiona inadequado suprimento sanguíneo para atender necessidades metabólicas tissulares, na presença de retorno venoso normal, ou fazê-lo somente com elevadas pressões de enchimento.

$\mathrm{Na}$ maioria das formas de IC, a redução do débito cardíaco é 
responsável pela inapropriada perfusão tecidual (IC com débito cardíaco reduzido). De início este comprometimento do débito cardíaco se manifesta durante o exercício, e com a progressão da doença ele diminui no esforço até ser observado sua redução no repouso. ${ }^{1}$

O mecanismo responsável pelos sintomas e os sinais clínicos podem ser decorrentes da disfunção sistólica, diastólica ou de ambas, acometendo um ou ambos os ventrículos. Nos adultos, em aproximadamente $60 \%$ dos casos, está associada à disfunção ventricular esquerda sistólica e nos restantes $40 \%$ à disfunção diastólica, devendo ser realçado que esta última vem sendo mais observada com o aumento da expectativa de vida da população. ${ }^{1}$

A estratificação de pacientes com IC é baseada em dados históricos, exames físicos e investigação laboratorial e permite ao profissional de saúde avaliar o momento evolutivo da doença em que o paciente se encontra; avaliar a qualidade de vida e o prognóstico, estabelecendo prioridades e tratamento terapêutico adequado. ${ }^{1}$

A definição da etiologia é a etapa fundamental da avaliação dos pacientes com IC, contribui para a avaliação do prognóstico e pode influenciar a terapia. Na avaliação prognóstica é importante não só para o paciente se programar para o seu futuro e de seus familiares, quanto para seu médico, que deve estar atento ao melhor momento para a indicação de terapias de maior custo como ressincronizadores, desfibriladores, dispositivos mecânicos e transplante cardíaco. ${ }^{3,4,5}$ 
O prognóstico em longo prazo ainda é ruim, com sobrevida de cinco anos menor do que $50 \%$. Aproximadamente $50 \%$ da população com IC são sintomáticos e apresentam QV reduzida, entretanto, os fatores fisiológicos tais como a fração de ventricular esquerda e a tolerância ao exercício têm sido medidas tradicionais como preditoras da descompensação da IC e da mortalidade. $6,7,8,9,4$

No Brasil no ano de 2007, as doenças cardiovasculares representaram a terceira causa de internações no SUS, com 1.156.136 hospitalizações. A IC é a causa mais freqüente de internação por doença cardiovascular. Em 2006, a IC e cardiopatias associadas à IC foram responsáveis por $6,3 \%$ dos óbitos no Estado de São Paulo, estado mais populoso do Brasil com 41.654.020 habitantes. Em 2007, a IC foi responsável por $2,6 \%$ das hospitalizações e por $6 \%$ dos óbitos registrados pelo SUS-MS no Brasil, consumindo $3 \%$ do total de recursos utilizados para atender todas as internações realizadas pelo sistema. Os dados sobre atendimentos ambulatoriais e hospitalares por IC neste cenário não estão disponíveis. $^{10}$

No Brasil, a principal etiologia da IC é a cardiopatia isquêmica crônica associada à hipertensão arterial. Em determinadas regiões geográficas do país e em áreas de baixas condições sócio-econômicas, ainda existem formas de IC associadas endemicamente à doença de Chagas. A endomiocardiofibrose e a cardiopatia valvular reumática crônica, ainda têm importância epidemiológica em nosso meio. ${ }^{11}$ 
Nos pacientes com mais de 60 anos, a IC é a principal causa de internação e se torna um grave problema de saúde pública em todo o mundo. Além dos altos custos hospitalares e de atendimentos de emergência, a IC provoca uma sensível perda da $Q V$, resultando, muitas vezes em aposentadorias precoces e em altos custos econômicos para o país. ${ }^{12}$

\subsection{COMPLEXIDADE DO TRATAMENTO NA INSUFICIÊNCIA CARDÍACA}

Os principais objetivos do tratamento na IC são aliviar os sintomas, melhorar a capacidade funcional ou a capacidade física e prolongar o tempo de vida.

Apesar da evolução terapêutica na IC, nas formas mais avançadas a IC continua sendo uma síndrome de alta complexidade e alta mortalidade e preveni-la pode ser a melhor forma de reduzir a sua incidência.

Devido à alta complexidade do tratamento, a IC requer cuidados contínuos e gastos elevados para o tratamento ambulatorial e está associada com freqüente hospitalização, comprometimento funcional, e alta mortalidade ${ }^{13}$, com prognóstico reservado, iguais ou pior a vários tipos de neoplasias malignas. ${ }^{14,15}$

Considerando que a IC é uma síndrome complexa, seu tratamento pode necessitar de várias estratégias combinadas, baseadas em diferentes mecanismos fisiopatológicos. Assim o tratamento atual objetiva não só o 
alívio dos sintomas, mas também impedir ou retardar a progressão da disfunção ventricular, bem como reduzir a mortalidade. ${ }^{16,17,18,19}$

O tratamento da IC deve levar em consideração o status funcional do paciente e as manifestações clínicas presentes, o estágio da doença, presença de co-morbidades e modalidade predominante de IC. ${ }^{1}$

A maioria dos pacientes com IC responde ao tratamento clínico evoluindo com melhora da sobrevida, da capacidade funcional, dos sintomas e redução das taxas de hospitalização. Entretanto um grupo de pacientes com IC avançada persiste sintomático a despeito da terapia médica otimizada. Apesar da diminuição da mortalidade por IC na última década, esta ainda permanece muito elevada. ${ }^{20,21,22}$ Para essa população o suporte circulatório mecânico e o transplante cardíaco podem ser considerados. ${ }^{1}$

Para êxito do tratamento de pacientes com IC, além da instituição da terapia medicamentosa capaz de promover o alívio dos sintomas e a modificação na evolução da doença, outras medidas, como a educação e orientação de pacientes e seus cuidadores devem ser observadas para melhora da qualidade de vida e possivelmente da sobrevida. ${ }^{23,24}$

Torna-se importante ressaltar a importância do tratamento não farmacológico, que deixou de ser um simples complemento da farmacoterapia, tornou-se atualmente parte integrante indispensável da terapêutica da insuficiência cardíaca. A não aderência a estas medidas pode colocar em risco a eficácia do tratamento. É fundamental ressaltar a importância da aderência ao tratamento farmacológico e não 
farmacológico para o sucesso da terapêutica, que pode representar um desafio não menos sério que as inerentes a própria doença.

\subsection{IMPORTÃNCIA DA EDUCAÇÃO E MONITORIZAÇÃO}

A educação de pacientes com IC caracteriza-se por um processo de melhora dos seus conhecimentos e de suas habilidades que influenciam as atitudes requeridas do paciente para um comportamento ideal em saúde. É fundamental em tratamentos complexos com alterações do estilo de vida e importante impacto na sua QV e de seus familiares, necessitando de acompanhamento permanente da equipe de saúde. ${ }^{25}$

Com o aumento da complexidade do tratamento da IC, medicamentos adicionais de administração mais elaborada ou polifarmácia e procedimentos cirúrgicos de alto custo ou complexidade sugerem a utilização de clínicas de IC, onde a educação pode ser fundamental. As clinicas de IC podem beneficiar os pacientes quando acompanhadas de educação e monitorização, pois além de serem um modelo assistencial de baixo custo adequado às nossas condições sociais e econômicas, podem proporcionar uma melhora da QV. ${ }^{26,27,28,29}$

As clínicas de IC podem desenvolver programas de educação intensiva sobre IC e monitorização no seguimento, e têm como objetivo a educação do paciente para garantir a aderência ao tratamento farmacológico e não farmacológico, identificando precocemente sintomas, fatores 
relacionados com a descompensação, ou eventos passíveis de tratamento e prevenção, no ambiente extra-hospitalar. ${ }^{30}$

Em geral, a base profissional da clínica de IC e de seus programas, é constituída por médico cardiologista especialista em IC e enfermeira. A enfermeira coordena a rápida resposta às múltiplas solicitações e serviços para os outros membros da equipe, tais como orientação psicológica, nutricional, social, farmacológica, assim como a educação do paciente/família e monitorização contínua destas ações. ${ }^{31,32,33,34}$

Estudo realizado em centro terciário (Instituto do CoraçãoHCFMUSP), com educação e monitorização por telefone por equipe interdisciplinar em longo prazo, com inclusão de 350 pacientes com diagnóstico de IC em acompanhamento ambulatorial randomizados em grupo intervenção e controle, com seguimento médio de 2,4₫1,75 anos, demonstrou redução no número de hospitalizações e número de visitas em unidades de emergência e óbito, com melhora da qualidade de vida e aderência (REMADHE trial ). ${ }^{35}$

\subsection{QUALIDADE DE VIDA NA INSUFICIÊNCIA CARDÍACA.}

Ao mesmo tempo em que há um crescente desenvolvimento tecnológico da medicina e ciências afins, paradoxalmente surge a preocupação com o conceito de "qualidade de vida", apresentando-se dentro das ciências humanas e biológicas como uma valorização mais ampla dos parâmetros que controlam os sintomas, a diminuição da mortalidade ou 
aumento da expectativa de vida. A avaliação da QV tem sido incluída nos ensaios clínicos randomizados como a terceira dimensão a ser avaliada, além da eficácia (modificação da doença pelo efeito da droga) e da segurança (reação adversa a drogas). ${ }^{36}$

Estudos demonstram que ela aparece pela primeira vez no Index Medicus em 1985. ${ }^{37,38}$ Desde então a QV passou a ser pesquisada com maior freqüência na área da saúde nos últimos anos, devido a forte relação entre doença e QV.

A Organização Mundial de Saúde (OMS) (1974) define QV como “... percepção de sua posição na vida no contexto da cultura e sistema de valores no qual elas vivem e em relação as suas metas, expectativas, normas e conceitos". ${ }^{39}$ Dentro do contexto da saúde, a OMS foi a pioneira em reconhecer a importância das três dimensões: física, mental e social.

A idéia de QV foi pouco mencionada na literatura até 1975 , quando pesquisadores começam a descobrir atributos da QV, bem como os instrumentos que podem ser desenvolvidos para medi-los e a predizer as intervenções que podem ajudar a melhorar a QV. No Brasil, passou a ter significado e importância na literatura brasileira a partir de 1992.

A QV é mais do que uma descrição do estado de saúde do paciente, é uma reflexão de como o paciente percebe e reage ao seu estado de saúde e a outros aspectos não médicos de sua vida. Estas percepções e reações podem ser determinadas, solicitando-se aos pacientes a sua avaliação sobre a QV global e também, ressaltando-se a importância dos itens individuais que afetam a sua QV. Assim, saúde transformou-se em um conceito 
multidimensional, incorporando os diversos aspectos da vida como perspectiva de vida, relações e papéis sociais. ${ }^{40}$

A QV é uma nova medida de mensurar a evolução dos efeitos das estratégias do tratamento no curso da doença. A QV está sendo considerada como uma nova dimensão da avaliação dos resultados de tratamentos, ao lado de parâmetros tradicionalmente utilizados como o controle de sintomas, índice de mortalidade e o aumento da expectativa de vida. ${ }^{41}$

Com o intuito de avaliar a QV em pacientes com IC, autores desenvolveram um instrumento específico, o Minnessota Living With Heart Failure Questionnaire (MLWHFQ). Esse instrumento baseia-se principalmente no quanto a doença, particularmente a IC, interfere na QV do paciente. ${ }^{42} \mathrm{O}$ MLWHFQ é o mais reconhecido instrumento específico para a IC, engloba os dois maiores domínios: as dimensões físicas e emocionais do ser humano, além de questões adicionais. ${ }^{43}$ Estudos anteriores demonstraram que as suas propriedades psicométricas são confiáveis e validadas. $44,45,46,47$

Em revisão da literatura realizada recentemente, o MLWHFQ foi o instrumento específico mais utilizado em pacientes com insuficiência cardíaca. $^{48}$

Estudos retratam que uma importante meta da terapêutica médica para pacientes com IC é reconhecer como os pacientes sentem e como desempenham as suas funções durante as suas atividades diárias, considera-se também que por meio da avaliação da QV é possível estabelecer o real estado de limitação da sua vida. ${ }^{49}$ Assim, o objetivo do 
tratamento na IC é também melhorar a QV seguida pelo aumento da sobrevida. ${ }^{50}$ A esse respeito, autores indicam que o conhecimento dos resultados da $\mathrm{QV}$ relacionada à saúde na $\mathrm{IC}$ severa pode orientar as decisões e ser útil na avaliação de novas terapias. ${ }^{51}$

Atualmente observa-se uma preocupação na comunidade científica em identificar os determinantes de QV, especialmente em situações de doenças crônicas, que busquem avaliar as implicações da doença no estilo de vida. Nas doenças crônicas, a IC constitui uma das principais causas de incapacidade, principalmente no que se refere à auto limitação da atividade física. Esta limitação está intimamente relacionada com as atividades diárias, com a sua QV e as mudanças do estilo de vida impostas pela doença. ${ }^{52}$

Tem-se reconhecido que os pacientes com IC crônica têm duas metas principais: melhorar a QV e prolongar a vida. Frequentemente os pacientes dão maior importância a qualidade de vida. Nos ambulatórios e especialmente nas clínicas de insuficiência cardíaca a avaliação da QV de pacientes com IC tem-se revelado cada vez mais importante, particularmente no que abrange os aspectos relacionados à doença, e o quanto ela interfere em sua vida. ${ }^{53}$

A assistência ao paciente deve estar centrada no atendimento das necessidades humanas básicas, de forma sistemática e individualizada, tornando-se fundamental que os profissionais da área de saúde realizem a avaliação da QV no intuito de resgatar as dimensões em prejuízo e potencializar as que se encontram preservadas. $O$ detalhamento do comprometimento das dimensões da QV tem o potencial de identificar 
elementos que subsidiem as ações dos profissionais buscando resultados positivos e aprimorando os procedimentos terapêuticos.

Diante desse contexto, nesse estudo optamos pela utilização do instrumento MLWHFQ que focaliza a percepção do paciente sobre os efeitos da IC em suas vidas, tendo-se em vista que o mesmo possui a adaptação transcultural para a língua portuguesa do Brasil desde 2001 e validação de suas propriedades psicométricas em 2009. ${ }^{46,47}$

\subsection{HIPÓTESE DO ESTUDO}

Neste estudo testamos a hipótese de que um programa seqüencial de educação e monitorização, contínuo e à longo prazo, baseado em monitorização telefônica contínua e atividades educativas repetitivas, pode beneficiar de maneira não uniforme os componentes da QV em pacientes com IC em seguimento ambulatorial. A análise dos efeitos deste programa na QV e de seus componentes pode determinar o desenvolvimento de novas estratégias, e conseqüentemente de possíveis novas intervenções terapêuticas que beneficiem o paciente. 
2. Objetivos 


\section{OBJETIVOS}

\section{1. OBJETIVO PRIMÁRIO}

Demonstrar que um programa seqüencial repetitivo de educação e monitorização telefônica com características desenvolvidas para o nosso meio cultural, contínuo e de longo prazo, pode beneficiar de maneira não uniforme os componentes da qualidade de vida de pacientes com IC em seguimento ambulatorial.

\section{2. OBJETIVOS SECUNDÁRIOS}

2.2.1. Comparar os aspectos específicos dos componentes da qualidade de vida dos grupos ntervenção e controle.

2.2.2. Avaliar a influência do sexo na qualidade de vida entre os grupos intervenção e controle.

2.2.3. Avaliar a influência da qualidade de vida na sobrevivência.

2.2.3. Avaliar os fatores relacionados á sobrevivência e a incidência de eventos. 


\section{Casuística e Métodos}




\section{CASUÍSTICA E MÉTODOS}

\subsection{POPULAÇÃO}

Este estudo é uma sub-análise de uma extensão REMADHE "trial" (Repetitive Education and Monitoring for Adherence in Heart Failure) desenvolvido no Instituto do Coração - HCFMUSP na unidade de insuficiência cardíaca, que incluiu pacientes com IC em seguimento ambulatorial e cuidados cardiológicos especializados em insuficiência cardíaca dentro de uma clínica de IC.

O critério de elegibilidade foi de pacientes com seguimento ambulatorial e com diagnóstico de IC crônica com pelo menos seis meses de duração.

\section{Critérios de inclusão:}

- Pacientes em acompanhamento no ambulatório de IC e transplante cardíaco, que preenchessem os critérios diagnósticos de Framingham modificados para IC. ${ }^{47,48}$

\section{Critérios de exclusão:}

- Pacientes com incapacidade de comparecer às sessões educacionais e/ou de serem submetidos à monitorização por:

- Falta de condições para transporte do paciente e acesso ao hospital;

- Residência fora do município;

-Barreiras sociais e de comunicação (ausência de contato telefônico); 
-Infarto do miocárdio; angina instável, cirurgia ou angioplastia cardíaca nos seis meses que antecedem a randomização;

- Pacientes hospitalizados ou com alta hospitalar recente;

- Doença grave renal/hepática/neurológica/pulmonar ou sistêmica com potencial para modificar a interpretação dos resultados e a sobrevida esperada e que cause limitações para comparecer às sessões educacionais;

- Procedimento que poderia influenciar o seguimento;

- Mulheres com potencial para gravidez ou gestantes.

\subsection{DINÃMICA DO ESTUDO}

Foram avaliados 639 pacientes, no período de outubro de 1999 a janeiro de 2006, sendo incluídos 412, com seguimento e acompanhamento até 20 de março de 2009. (Figura 1)

Os pacientes foram submetidos a um programa prospectivo de educação e monitorização repetitiva contínua em um único centro, aberto, com randomização simples para comparar grupo intervenção versus controle (Estudo REMADHE). ${ }^{35}$ A sub-análise das dimensões da QV foi retrospectiva. Após a randomização, os pacientes foram informados quanto à finalidade do estudo e foi solicitado o de acordo com a assinatura do termo de consentimento informado.

O primeiro paciente foi randomizado em 05 de outubro de 1999 e o último em 08 de fevereiro de 2006. A randomização foi realizada por uma lista gerada por computador, com relação de 2:1, foi distribuída em blocos de 3 que incluem 2 intervenções e 1 controle em paralelo. A ordem de 
intervenções e de controle dentro de cada bloco foi atribuída aleatoriamente. Para evitar a dedução da alocação seguinte da intervenção e para a organização dos grupos, os pesquisadores eram cegos quanto ao tamanho dos blocos. A cada randomização incluiu-se um número de pacientes múltiplo de três com pelo menos 15 participantes elegíveis, à exceção do último grupo. Para o não reconhecimento da alocação foram utilizados caixas seqüenciais, numeradas, opacas, e seladas. As caixas foram abertas seqüencialmente e somente depois os nomes dos participantes foram escritos na caixa apropriada. A enfermeira envolvida na educação/ monitorização registrou os pacientes e atribuiu os participantes a seus grupos. Os participantes, a enfermeira e a equipe multidisciplinar não foram cegos quanto aos grupos formados. Aqueles que avaliam os resultados foram cegos quanto aos grupos formados e aos valores atribuídos. (Figura 2)

A equipe de pesquisa não participou em decisões da intervenção. Dados sobre hospitalizações, mortes, formas de morte, necessidade de atendimentos de emergência, procedimentos, e eventos foram obtidos através de relatos dos pacientes coletados em visitas médicas, de familiares, de ligações telefônicas, e de revisão de prontuários. No momento da inclusão e em intervalos de 6 até 24 meses, e após anualmente, foram aplicados os escores dos questionários de QV para a insuficiência cardíaca (MLWHFQ) e aderência.

Tanto os pacientes do grupo intervenção como os do controle tiveram seguimento médico habitual da instituição, executado durante o 
período do estudo pela mesma equipe ambulatorial de cardiologistas, que não foram informados da randomização. O intervalo programado entre avaliações ambulatoriais rotineiras foi de 3-4 meses. Também, a equipe ambulatorial não participou de nenhuma etapa do estudo. A equipe ambulatorial de cardiologistas foi orientada a seguir as Diretrizes Brasileiras para tratamento de insuficiência cardíaca. Consultas ambulatoriais extras foram realizadas dependendo das necessidades de cada paciente e da decisão do cardiologista. O enfermeiro foi o centralizador da equipe interdisciplinar, e responsável pela orientação/educação, monitorizando os pacientes por meio de contatos telefônicos mensais e/ou quinzenais, consultas de enfermagem e encaminhamento aos profissionais específicos, quando necessário. (Tabela 1)

Na presença de complicações ou agravamento da condição clínica, o enfermeiro indicava ao paciente uma visita de comparecimento não planejada ao cardiologista. Não ocorreu contato telefônico ou por qualquer outra tecnologia entre a equipe de cardiologistas e os pacientes, tal como a monitorização eletrônica da pressão arterial sanguínea, eletrocardiograma, oxímetro de pulso, telemetria ou internet.

As entrevistas foram realizadas pelo pesquisador e alunos da iniciação científica do curso de graduação em enfermagem do programa bolsa COSEAS. Os alunos receberam orientação e treinamento para o desenvolvimento destas atividades, tendo sido utilizado o mesmo padrão e interpretação das respostas. 
Entrevistas ocorreram por meio de contatos telefônicos e pessoalmente, quando o paciente comparecia ao laboratório de insuficiência cardíaca, em intervalos mensais, e com intervalos menores na presença de alterações que necessitassem de avaliação e/ou intervenção no tratamento farmacológico e não farmacológico.

O estudo foi aprovado pela Comissão de Ética para Análise de Projetos de Pesquisa (CAPPesq ) do Hospital das Clínicas da Faculdade de Medicina de São Paulo.

Foi observado o anonimato e a privacidade dos pacientes, respeitando os aspectos éticos que envolvem a pesquisa em seres humanos conforme as Diretrizes e Normas da Resolução n 196/1996 do Conselho Nacional de Saúde - Ministério que regulamenta pesquisas envolvendo seres humanos. 
Figura 1. Desenho do Estudo

Pts avaliados para o Programa de Educação e Monitorização

$N=639$

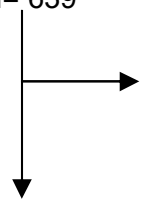

Critérios de Exclusão (n=227)

Pts Randomizados

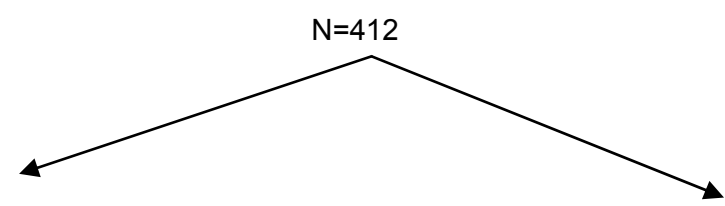

Grupo Intervenção

$\mathrm{N}=275$

Grupo Controle

$\mathrm{N}=137$

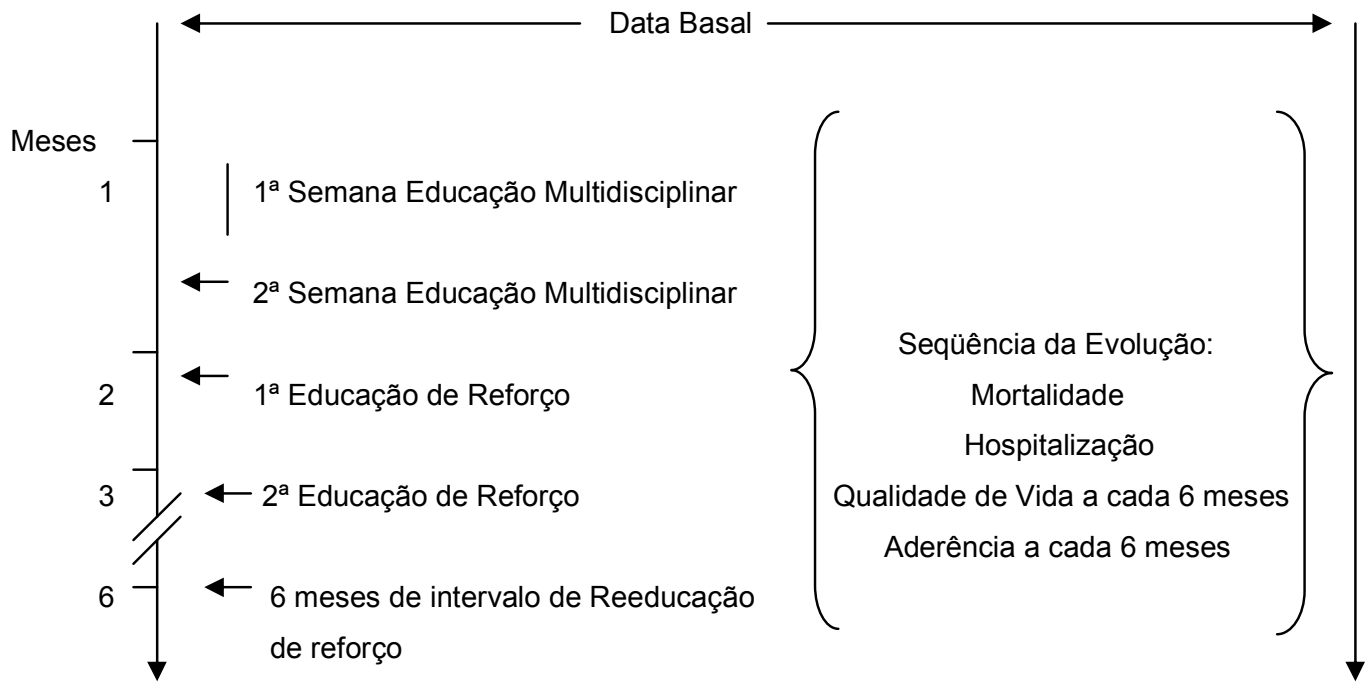


Figura 2. Fluxograma do Estudo

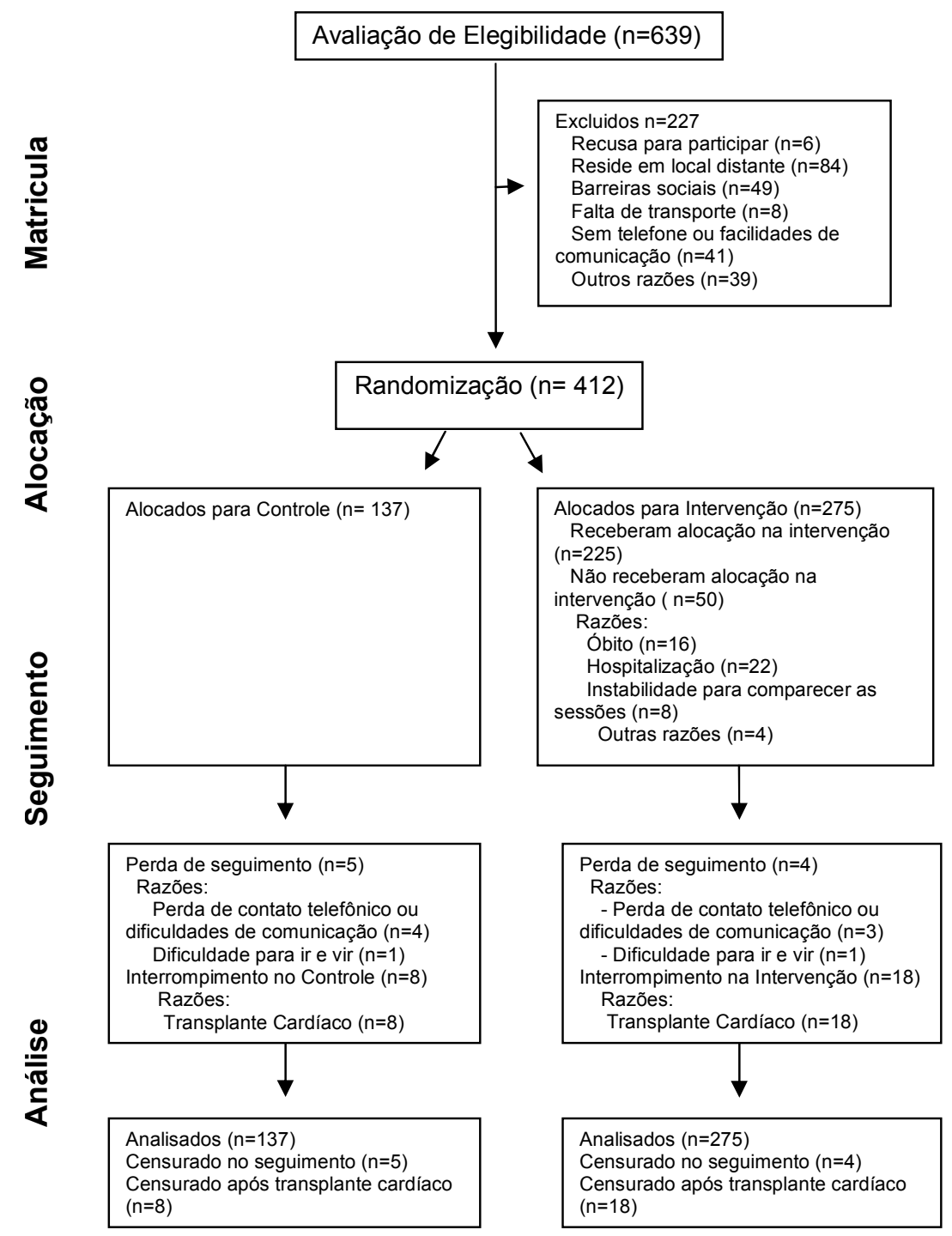




\title{
Tabela 1 - Programa de atividades nas Intervenções
}

\author{
Intervenção e Classes de Educação \\ Intervenções \\ Duração: 30 minutos com alguns componentes interdisciplinares \\ Pessoal: enfermeiros, farmacêuticos, assistentes sociais, nutricionistas e psicólogos. \\ Projeto: pessoalmente incluindo todos os pacientes randomizados do grupo intervenção \\ Programação: recente após a randomização \\ Educação de Classes: \\ Duração: 60 minutos \\ Equipe multidisciplinar (enfermeiros, farmacêuticos, assistentes sociais, odontologistas, \\ nutricionistas, psicólogos) \\ Projeto: grupo de classes incluindo todos os pacientes randomizados do grupo intervenção. \\ Programação: recente após as randomizações (quatro vezes) e durante o seguimento. \\ Intervalo: uma semana entre a $1^{\mathrm{a}}$ e $2^{\mathrm{a}}$; um mês entre o $2^{\circ}, 3^{\circ}\left(1^{\mathrm{a}}\right.$ reunião de reforço) \\ e $4^{\circ}$ ( $2^{\mathrm{a}}$ reunião de reforço); e após com intervalo de seis meses. \\ Conteúdo apresentado pelo entrevistador na educação de classes: \\ Relato de princípios básicos da Insuficiência Cardíaca (IC). \\ Causas fitopatológicas da IC, causas de sobrecarga de volume. \\ Bases fundamentais no tratamento farmacológico e não farmacológico. \\ Importância da aderência aos medicamentos, mecanismo de ação e efeitos colaterais. \\ Restrição hídrica, dieta hipossódica, (a menos que, apresente contra indicação médica). \\ Cuidados com a insuficiência cardíaca, monitorização diária do peso corporal. \\ Abolir o álcool, fumo e medicamentos antiflamatórios. \\ Educação quanto a atividade física, trabalho e atividades físicas diárias. \\ Sinais de descompensação e piora da IC. \\ Sinais e sintomas de reações de piora da IC (especialmente, sincope, taquicardia, hemoptise, \\ ascite, edema, febre, piora da dispnéia, dor torácica, dispnéia paroxística noturna, tosse \\ noturna, ortopnéia, oligura, anorexia, dor abdominal). \\ Educação e Orientação sobre o que fazer se os sintomas se agravarem ou inicio de um novo \\ sintoma. \\ Potenciais medicamentos que podem causar pior a IC (estrógeno, corticóide, antagonistas de \\ cálcio, antiarrítmicos, e outros). \\ Monitorização \\ Contatos telefônicos. \\ Inicio: após a $2^{\mathrm{a}}$ reunião educativa. \\ Intervalos: programado em uma freqüência de 14 dias (mais ou menos e concordando \\ aproximadamente com a decisão da enfermeira dependendo da necessidade). \\ Pessoal: enfermeira treinada no programa de gerência da IC. \\ Indice: questionário Minnesota Living with Heart Failure Questionnaire. \\ Objetivo: reforço na educação. \\ Monitorização da aderência e complicações, sinais e sintomas de piora da IC e \\ mecanismos de auto controle. \\ Ajuste da dose do diurético pelo enfermeiro conforme necessidade após avaliação \\ clínica de sintomas e sinais de piora da ou melhora da IC.
}




\subsection{QUALIDADE DE VIDA}

A qualidade de vida foi avaliada pelo questionário MLWHFQ específico para pacientes com IC. Este instrumento foi aplicado nos pacientes randomizados no período basal e durante o seguimento na clínica de insuficiência cardíaca por meio de entrevista direta e individualizada. (Anexo 1)

O instrumento MLWHF é um questionário composto de 21 questões relativas a limitações que freqüentemente estão associadas com o grau de impedimento que a IC possa ocasionar nos pacientes e permite uma autoavaliação do impacto sobre os componentes físico, psicológico e social da $\mathrm{IC} ;{ }^{34}$ sempre esclarecendo os pacientes que eles deverão considerar o último mês para responder ao questionário. A escala de respostas para cada questão varia de 0 (zero) a 5 (cinco), sendo que o "0" representa sem limitações e o "5" limitação máxima. Baseado em estudos prévios, um escore de dimensão física foi calculado como a soma de oito questões (de números 2, 3, 4, 5, 6, 7, 12 e 13) que estão altamente inter-relacionadas com dispnéia e fadiga. Similarmente, o escore da dimensão emocional é composto de cinco questões (números 17, 18, 19, 20 e 21) altamente interrelacionadas. As demais questões (números 1, 8, 9, 10, 11, 14, 15 e 16) somadas às dimensões físicas e emocionais, formam o escore total, aplicado por meio de entrevista direta no tempo de 5 a 10 minutos. Para garantir a melhor compreensão por todos os participantes, cada questão foi lida e explicada pelo avaliador, tomando-se cuidado em evitar a indução das 
respostas, que foram registradas pelo avaliador. Para maior homogeneidade das respostas, os pacientes foram entrevistados isoladamente. Foram analisados os 21 itens do instrumento. (Tabela 2) 
Tabela 2 - Dimensões da qualidade de vida

\section{Dimensão física (DF)}

2. Obrigando você a sentar ou deitar para descansar durante o dia.

3. Tornando sua caminhada e subida de escadas difíceis.

4. Tornando seu trabalho doméstico difícil.

5. Tornando suas saídas de casa difíceis.

6. Tornando difícil dormir bem à noite.

7. Tornando seus relacionamentos ou atividades com familiares e amigos difíceis.

12. Causando falta de ar.

13. Deixando você cansado, fatigado ou com pouca energia.

\section{Dimensão Emocional (DE)}

17. Fazendo você sentir-se um peso para familiares e amigos.

18. Fazendo você sentir uma falta de autocontrole na sua vida.

19. Fazendo você se preocupar.

20. Tornando difícil você concentrar-se ou lembrar-se das coisas.

21. Fazendo você sentir-se deprimido.

\section{Questões que somadas as DF e DE formam o escore total}

1. Causou inchaço em seus tornozelos e pernas.

8. Tornando seu trabalho para ganhar a vida difícil.

9. Tornando seus passatempos, esportes e diversão difíceis.

10. Tornando sua atividade sexual difícil.

11. Fazendo você comer menos as comidas que você gosta.

14. Obrigando você a ficar hospitalizado.

15. Fazendo você gastar dinheiro com cuidados médicos.

16. Causando a você efeitos colaterais das medicações 


\subsection{ANÁLISE ESTATÍSTICA}

Para a análise das grandezas específicas (variáveis), realizou-se a estatística descritiva das características quantitativas: média (M), desvio padrão (DP), e número de casos (N). Calcularam-se, também as variações relativas $(\Delta \%)$ e, na impossibilidade desta, as variações absolutas $(\Delta)$ entre os resultados do seguimento seqüencial de cada variável.

$\mathrm{Na}$ comparação das características basais dos grupos controle e intervenção foram empregados os testes $t$ de Student para valores não pareados e o teste exato de Fisher.

As curvas de sobrevivência e livre de eventos foram calculadas pelo método de Kaplan-Meier e o teste de log-rank (Mantel-Cox) foi empregado na comparação entre as curvas. Para análise uni variada e multi variada de fatores associados ao prognóstico utilizou-se da regressão proporcional de Cox.

A mensuração da QV foi realizada antes da intervenção, após seis meses e anualmente. Para a análise estatística dos dados referentes à qualidade de vida, foi utilizado a analise de variância de duplo fator, foi utilizado o corte no tempo de 72 meses, pois foi nesta faixa que se concentrou a maior proporção de pacientes com tempo de seguimento suficiente para a análise; uma pequena proporção de paciente possuía tempo de seguimento suficientemente longo para realizar análise de seguimento aos 108 meses, já que a última inclusão ocorreu em 08/02/2006. 


\section{Resultados}




\section{RESULTADOS}

\section{Dados Demográficos}

As características demográficas dos dados iniciais dos grupos controle e intervenção foram similares. A média de seguimento foi de $3,6 \pm 2,2$ anos para todos os pacientes, $3,4 \pm 2,3$ anos para o grupo controle e $3,7 \pm 2,2$ anos para o grupo intervenção $(p=n s)$. Cinco pacientes $(3,6 \%)$ do grupo controle e quatro pacientes $(1,4 \%)$ do grupo intervenção perderam o seguimento. O período de tempo entre a primeira randomização e a análise de resultado foi de 3454 dias; o tempo entre a última randomização e a análise de resultado foi de 1136 dias. O número de contatos telefônicos para monitorização foi de 21,6 \pm 12 por paciente. Para a análise estatística dos dados referentes à qualidade de vida, foi utilizado o corte no tempo de 72 meses, pois foi nesta faixa que se concentrou a maior proporção de pacientes com tempo de seguimento suficiente para a análise; uma pequena proporção de paciente possuía tempo de seguimento suficientemente longo para realizar análise de seguimento aos 108 meses, já que a última inclusão ocorreu em 18/01/2006.

As características dos pacientes avaliados estão apresentadas na tabela 3 . 
Tabela 3 - Características Basais dos Grupos Controle e Intervenção

\begin{tabular}{|c|c|c|c|}
\hline VARIÁVEL & CONTROLE N $=137$ & INTERVENÇÃO = 275 & $\mathrm{P}$ \\
\hline IDADE (ANOS) & $51 \pm 12$ & $51 \pm 11$ & NS \\
\hline GÊNERO MASC, (\%) & $70 \%$ & $68 \%$ & NS \\
\hline RAÇA: BRANCO/ NEGRO / MULATO / AMARELO (\%) & $59 / 22 / 19 / 1$ & $50 / 28 / 21 / 0,4$ & NS \\
\hline RADIOISÓTOPOS FEVE (\%) & $30 \pm 11$ & $29 \pm 11$ & NS \\
\hline RADIOISOTOPOS FEVD (\%) & $32 \pm 10$ & $32 \pm 10$ & NS \\
\hline DDVE (CM ) NO ECOCARDIOGRAMA & $7 \pm 1$ & $7 \pm 1$ & NS \\
\hline IMC (ÍNDICE DE MASSA CORPOREA) & $26,8 \pm 4,7$ & $26,3 \pm 5$ & NS \\
\hline NYHA, I/II/III/IV (\%) & 23/34/29/14 & 19/45/25/11 & NS \\
\hline IC SITÓLICA (FEVE $\leq 45 \%)$ & $80 \%$ & $81,6 \%$ & \\
\hline BLOQUEIO DE RAMOĒESUERDO & $14,5 \%$ & $19,7 \%$ & NS \\
\hline CAUSAS (\%) & & & NS \\
\hline ISQUÊMICA/ ALCOÓLICA/ CHAGÁSICA/ & $22 / 8 / 21$ & $28 / 4 / 16$ & \\
\hline HIPERTENSIVA/ IDIOPÁTICA/VALVAR/ OUTRAS & $22 / 10 / 3 / 14$ & $18 / 17 / 3 / 14$ & \\
\hline FIBRILAÇÃO ATRIAL OU FLUTTER & $14,5 \%$ & $9,8 \%$ & NS \\
\hline FARMACOTERAPIA -IC (\%) & & & NS \\
\hline AMIODARONA & $9,4 \%$ & $9,9 \%$ & \\
\hline AMLODIPINA & $6,8 \%$ & $3,9 \%$ & \\
\hline BRA & $21,4 \%$ & $7,7 \%$ & \\
\hline ACEI & $80 \%$ & $85 \%$ & \\
\hline B-BLOQUEADOR & $71 \%$ & $61 \%$ & \\
\hline ESPIRONOLACTONA & $58,1 \%$ & $53,6 \%$ & \\
\hline HIDRALAZINA & $9,4 \%$ & $3,4 \%$ & \\
\hline NitRATOS & $11,1 \%$ & $12,4 \%$ & \\
\hline DIGOXINA & $58,1 \%$ & $68,2 \%$ & \\
\hline DIURÉTICOS & $82,9 \%$ & $80,5 \%$ & \\
\hline FUROSEMIDA & $72 \%$ & $80,5 \%$ & \\
\hline ESTATINAS & $15,8 \%$ & $10,1 \%$ & \\
\hline \multicolumn{4}{|l|}{ OUTROS FARMACOTERÁPICOS(\%) } \\
\hline TERAPIA COM ANTICOAGULANTE ORAL & $9,4 \%$ & $12,4 \%$ & \\
\hline INSULINA & $0,9 \%$ & $3,4 \%$ & \\
\hline HORMÔNIO TIREOIDIANO & $5,1 \%$ & $3,4 \%$ & \\
\hline NIVEIS SÉRICOS DE SÓDIO, MEQ/L & $138 \pm 3$ & $139 \pm 3$ & NS \\
\hline$\%$ LEUCOCITOS & $7431 \pm 2687$ & $7464 \pm 1987$ & NS \\
\hline HEMOGLOBINA (G/L) & $14 \pm 2$ & $14 \pm 2$ & NS \\
\hline NIVEIS SÉRICOS CREATINA, MG/DL. & $1,4 \pm 1$ & $1,2 \pm 0,5$ & NS \\
\hline LDL COLESTEROL & $124 \pm 47$ & $125 \pm 38$ & NS \\
\hline HDL COLESTEROL & $45 \pm 15$ & $43 \pm 15$ & NS \\
\hline PICO DE VO2 (ML/KG/MIN) & $16,9 \pm 6,8$ & $17,5 \pm 6,4$ & NS \\
\hline NÍVEIS DE GLICOSE EM JEJUM & $109 \pm 30$ & $111 \pm 37$ & NS \\
\hline DURAÇÃO DE SINTOMAS DA IC (MESES) & $87 \pm 169$ & $84 \pm 155$ & NS \\
\hline № DE HOSPITALIZAÇÕES (PREVISTO EM 12 MESES) & $0,42 \pm 1,06$ & $0,39 \pm 1,09$ & NS \\
\hline RESINCRONIZAÇÃO & $2,6 \%$ & $3,9 \%$ & \\
\hline IMPLANTAÇÃO DE CARDIOVERSOR-DESFIBRILADOR & $0,85 \%$ & $0,43 \%$ & \\
\hline \multicolumn{4}{|l|}{ COMORBIDADES } \\
\hline DIABETES & $18,1 \%$ & $16,6 \%$ & \\
\hline DPOC & $3,4 \%$ & $1,28 \%$ & \\
\hline $\begin{array}{l}\text { PREVISÃO DE NÃO HOSPITALIZAÇÃO EM } 12 \text { MESES } \\
(\%)\end{array}$ & $82,9 \%$ & $82,4 \%$ & NS \\
\hline HIPOTIREOIDISMO & $7,7 \%$ & $7,3 \%$ & NS \\
\hline HIPERTIREOIDISMO & $0,8 \%$ & $1,7 \%$ & NS \\
\hline
\end{tabular}

FEVE, fração de ejeção de ventrículo esquerdo; FEVD, fração de ejeção de ventrículo direito; DDVE, diâmetro diastólico de ventrículo esquerdo; N.Y.H.A., New York Heart Association; IC, insuficiência cardíaca; BRA, bloqueador do receptor de angiotensina; ACEI, Inibidor de enzima de conversão de angiotensina; Pico Vo2, consumo máximo do oxigênio no exercício em esteira rolante; №., número de; DPOC, doença pulmonar obstrutiva crônica 
4.1. Qualidade de vida, número de atendimentos em unidades de emergência, número de internações e número de dias de internação.

$\mathrm{Na}$ analise da média da qualidade de vida global durante o seguimento, observamos melhora significativa do grupo intervenção $(p=$ 0,0005), assim como redução do número de atendimentos em emergência 2,0 vs. $6,1(p=0,002)$, do número de internações 0,8 vs. $2,3(p=0,007)$ e da média de dias de internação de 13,1 vs. $25,7(p=0,02)$ por paciente durante o seguimento respectivamente. A analise foi realizada com 108 meses de seguimento.

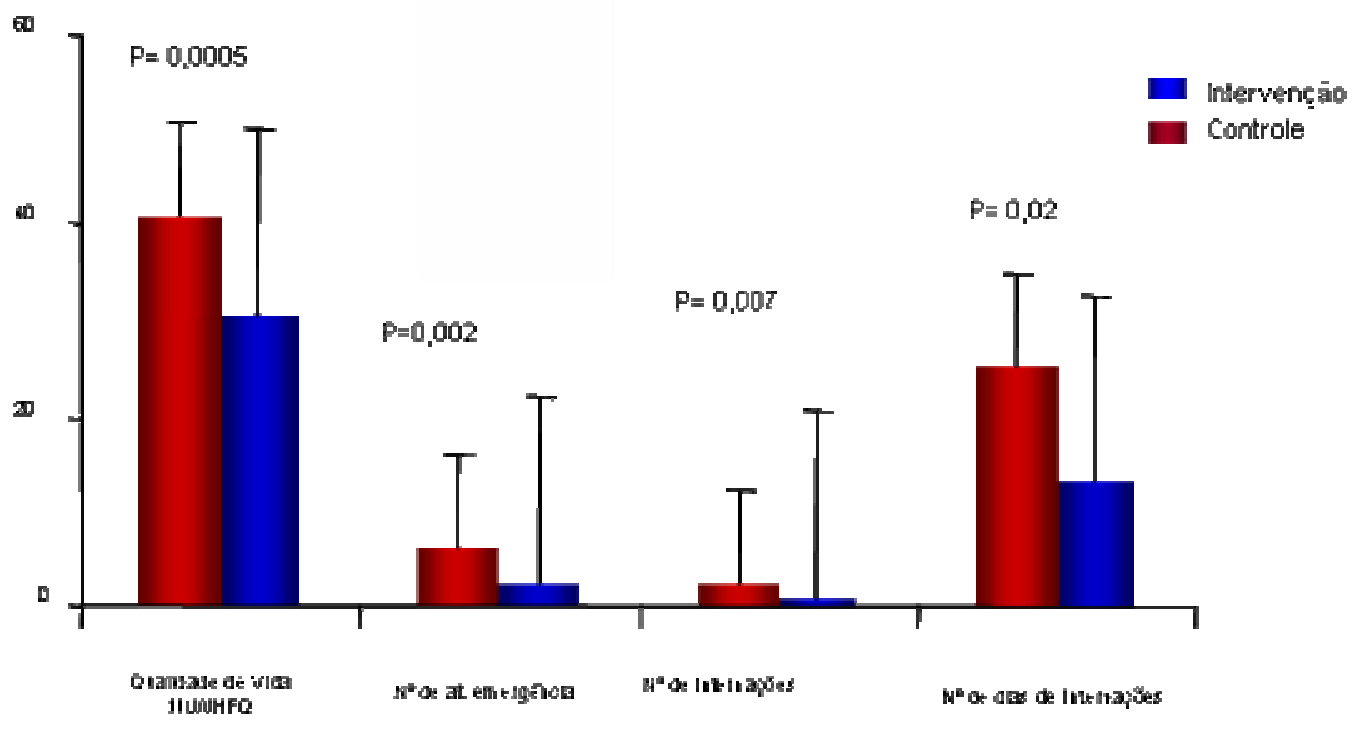

Figura 3. Qualidade de vida, número de atendimentos em unidades de emergência, número de internações e número de dias de internação. 


\subsection{Qualidade de Vida}

\subsubsection{Qualidade de Vida - Resultado global}

$\mathrm{Na}$ avaliação da qualidade de vida global houve uma variação ao longo do tempo e uma diferença significativa entre os grupos. O grupo intervenção melhorou ao longo do tempo de seguimento, enquanto que o grupo controle manteve valores semelhantes aos iniciais.

O valor de $\mathrm{p}$ em relação à diferença entre os grupos <0,0001; em relação à variação no tempo $<0,0001$ e houve interação significativa entre os dois fatores, intervenção e tempo $(p=0,0072)$. A analise foi realizada com 72 meses de seguimento.

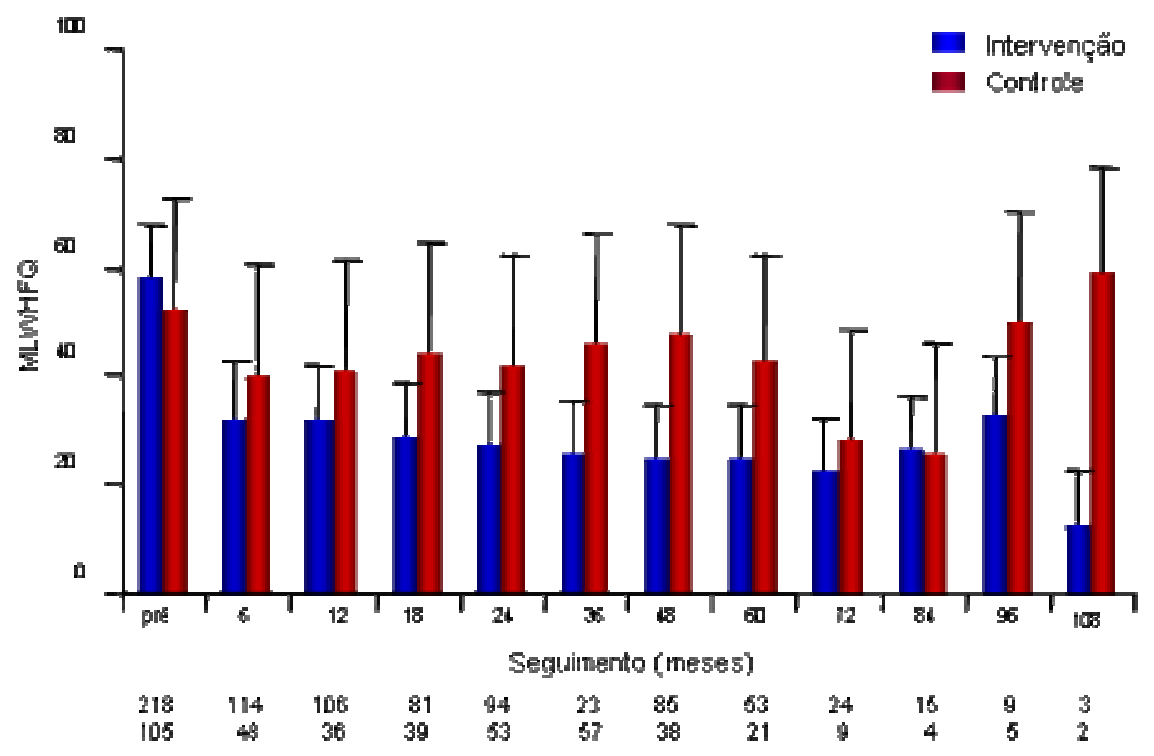

Figura 4. Qualidade de Vida - Resultado global. 


\subsubsection{Qualidade de Vida - Dimensão Física}

$\mathrm{Na}$ avaliação da dimensão física da qualidade de vida houve uma variação ao longo do tempo e uma diferença significativa entre os grupos. O grupo intervenção melhorou ao longo do tempo de seguimento, enquanto o grupo controle manteve valores semelhantes aos iniciais.

O valor de $p$ em relação à diferença entre os grupos foi <0,0001; em relação à variação tempo $<0,0001$ e houve interação significativa entre os dois fatores, intervenção e tempo $(p=0,0002)$. A analise foi realizada com 72 meses de seguimento.

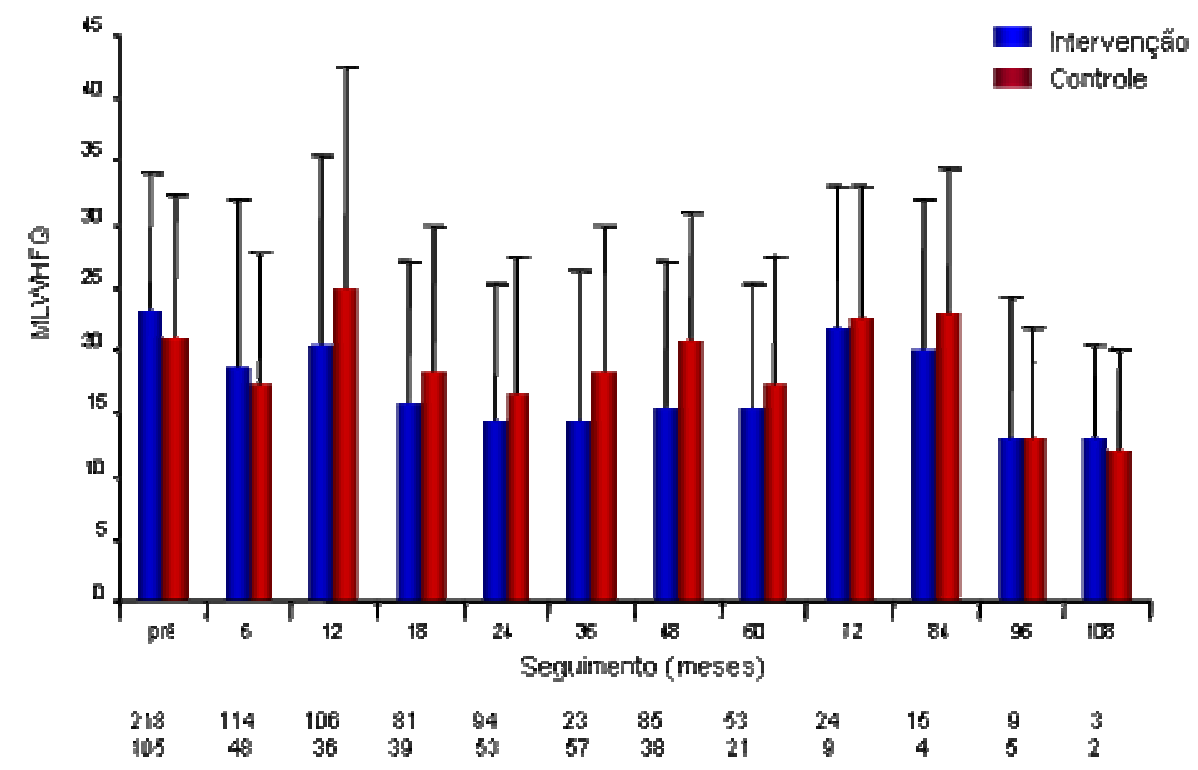

Figura 5. Qualidade de Vida - Dimensão Física 


\subsubsection{Qualidade de Vida - Dimensão Emocional}

$\mathrm{Na}$ avaliação da dimensão emocional da qualidade de vida houve uma variação ao longo do tempo e uma diferença significativa entre os grupos. O grupo intervenção melhorou ao longo do tempo de seguimento, enquanto o grupo controle manteve valores semelhantes aos iniciais.

O valor de $p$ em relação à diferença entre os grupos foi de $<0,0001$; em relação à variação tempo foi de $<0,0001$ e houve interação significativa entre os dois fatores, intervenção e tempo $p=0,0294$. A analise foi realizada com 72 meses de seguimento.

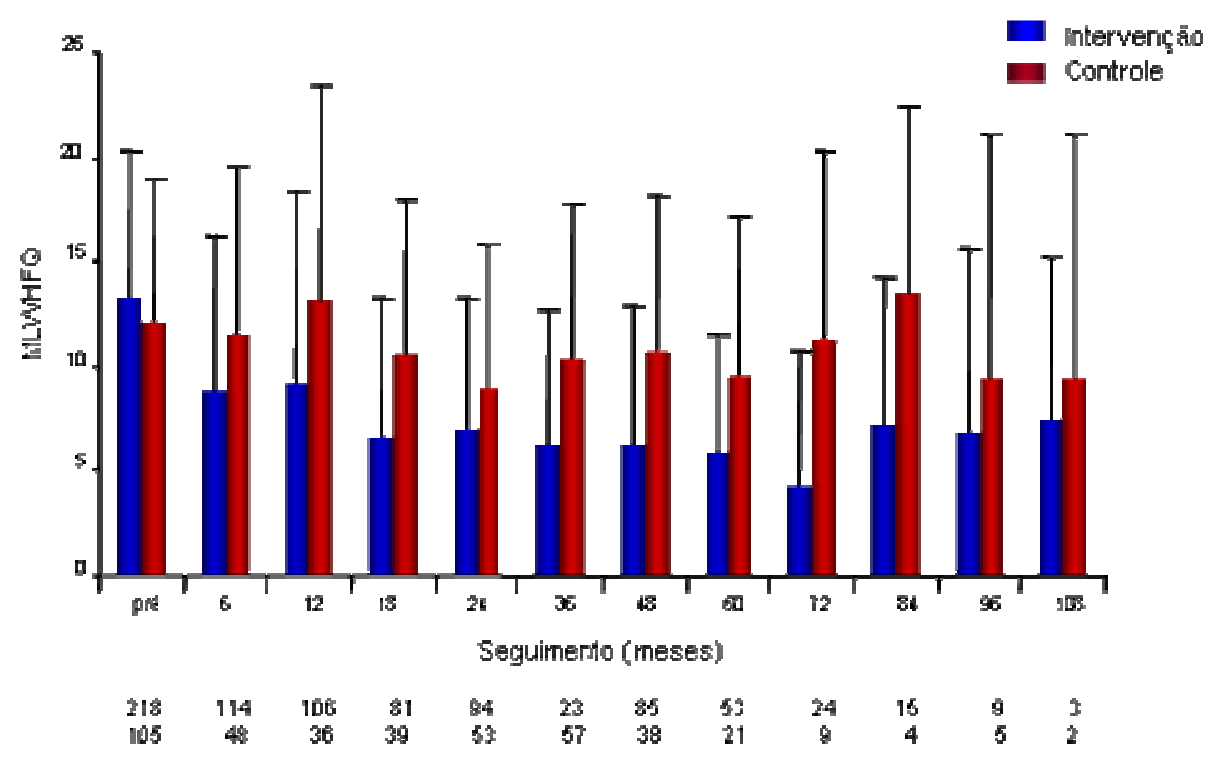

Figura 6. Qualidade de Vida - Dimensão Emocional 


\subsubsection{Qualidade de Vida - Demais Questões}

$\mathrm{Na}$ avaliação das demais questões da qualidade de vida houve uma variação ao longo do tempo e uma diferença significativa entre os grupos. $\mathrm{O}$ grupo intervenção melhorou ao longo do tempo de seguimento, enquanto que o grupo controle manteve valores semelhantes aos iniciais.

O valor de $\mathrm{p}$ em relação à diferença entre os grupos foi menor que 0,0001; em relação à variação tempo foi menor que 0,0001 e houve interação significativa entre os dois fatores, intervenção e tempo $(p=0,001)$. A analise foi realizada com 72 meses de seguimento.

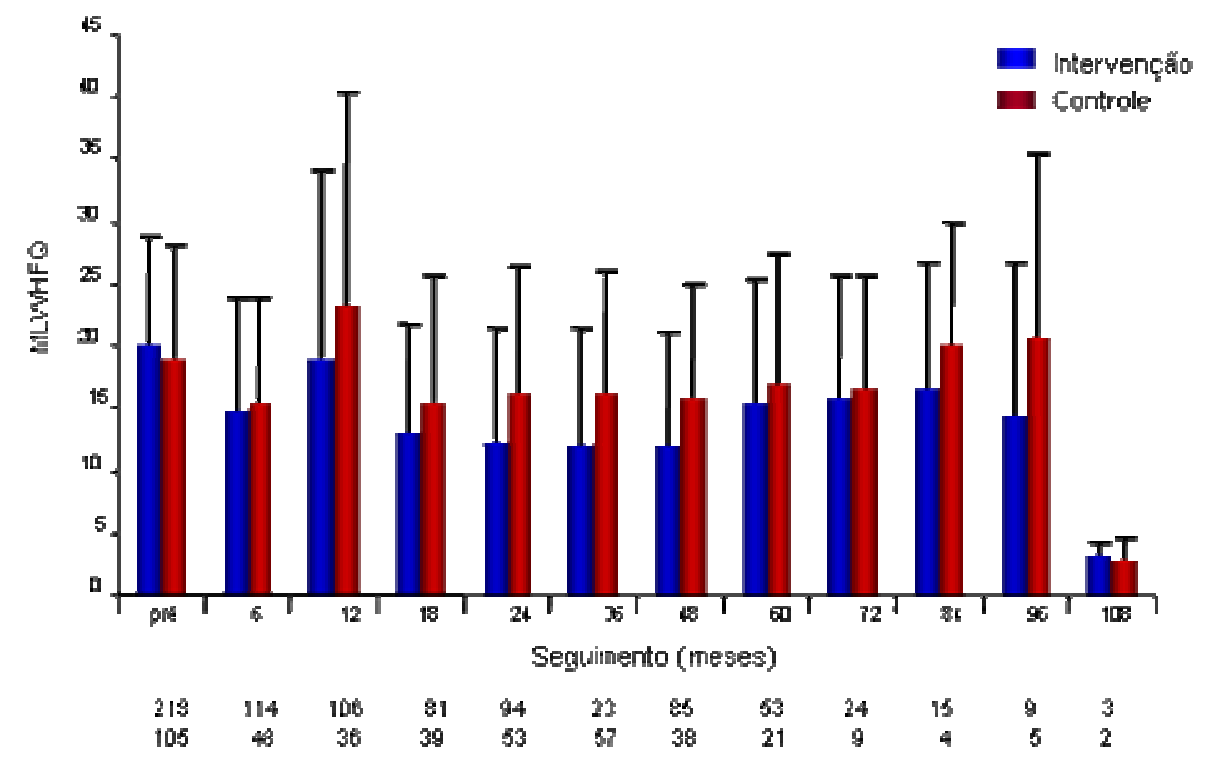

Figura 7. Qualidade de Vida - Demais Questões 


\subsection{Qualidade de Vida - Gêneros}

\subsubsection{Qualidade de Vida - Gênero Masculino}

$\mathrm{Na}$ avaliação da qualidade de vida global no gênero masculino houve uma variação ao longo do tempo e uma diferença significativa entre os grupos intervenção e controle. O grupo intervenção melhorou ao longo do tempo de seguimento, enquanto que o grupo controle manteve valores semelhantes aos iniciais.

O valor de $\mathrm{p}$ em relação à diferença entre os grupos foi menor que 0,0001; em relação à variação tempo foi menor que 0,0001 e houve interação significativa entre os dois fatores, intervenção e tempo $(p=0,0075)$.

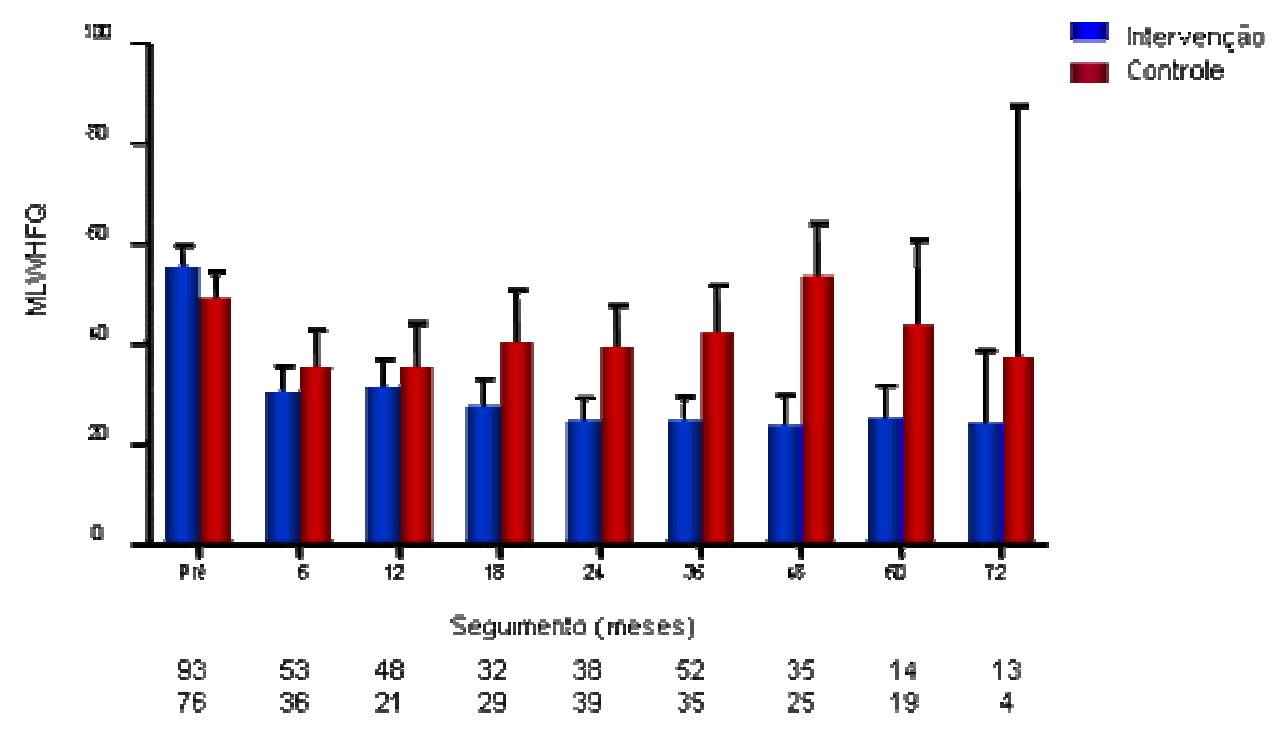

Figura 8. Qualidade de Vida - Resultado global - Gênero masculino 


\subsubsection{Qualidade de Vida - Gênero Feminino}

$\mathrm{Na}$ avaliação da qualidade de vida global no gênero feminino houve uma variação ao longo do tempo e uma diferença significativa entre os grupos. O grupo intervenção melhorou ao longo do tempo de seguimento, enquanto que o grupo controle manteve valores semelhantes aos iniciais durante grande período do seguimento.

O valor de $p$ em relação à diferença entre os grupos foi menor que 0,0001; em relação à variação tempo foi menor que 0,0001 e diferentemente do gênero masculino não ocorreu interação significativa entre os fatores intervenção e tempo $(p=0,2669)$.

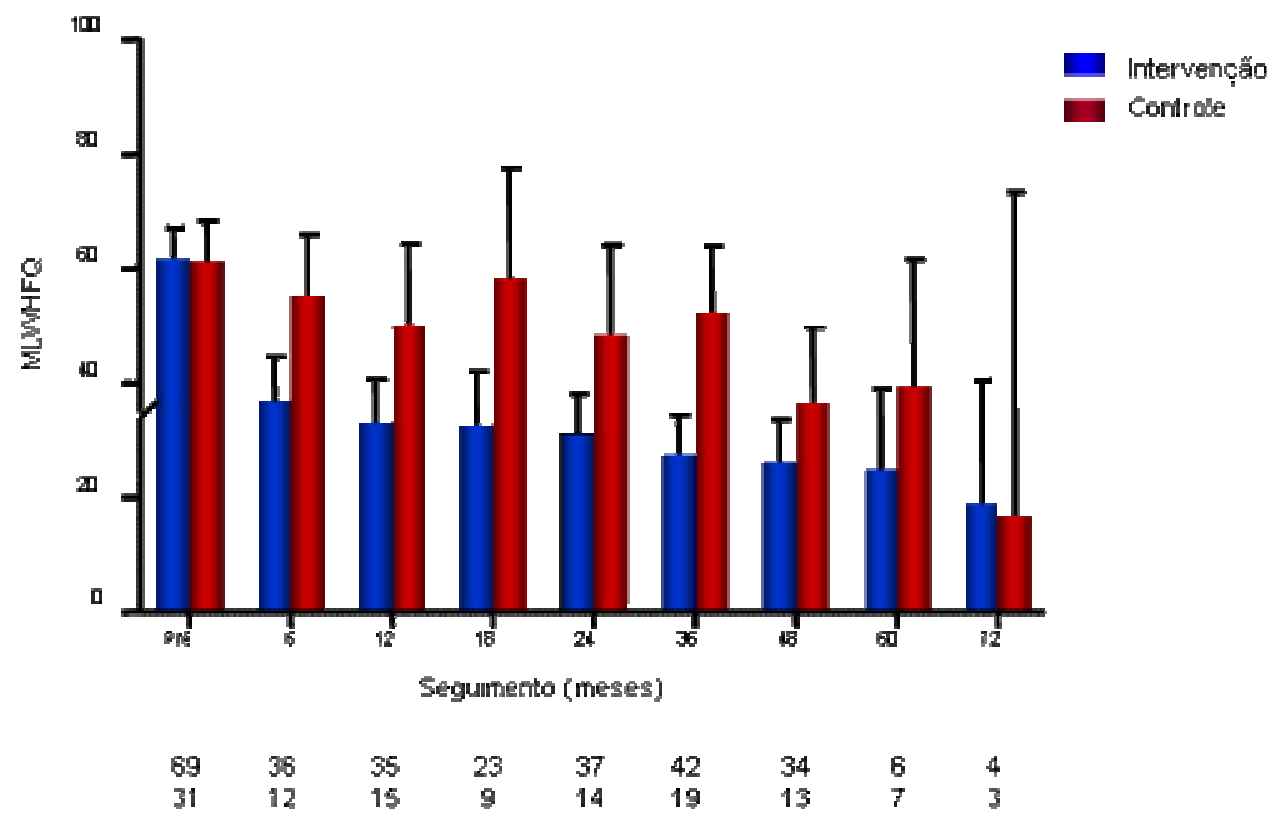

Figura 9. Qualidade de Vida- Resultado global - Gênero feminino. 


\subsection{Analise Univariada}

$\mathrm{Na}$ analise univariada os fatores que estiveram significativamente associados com sobrevida foram: gênero (sexo), idade, etiologia chagásica, qualidade de vida durante o seguimento, classe funcional e fração de ejeção de ventrículo esquerdo. (Tabela 4)

Tabela 4 - Analise univariada dos fatores associados à sobrevida

\begin{tabular}{lc}
\hline Variáveis & Significância $(\mathrm{p})$ \\
\hline Intervenção & 0,264 \\
Gênero & 0,034 \\
Idade & 0,002 \\
Etiologia chagásica & 0,007 \\
Qualidade de vida basal & 0,165 \\
Qualidade de vida após randomização & 0,006 \\
Aderência & 0,835 \\
Fração de ejeção de ventrículo esquerdo & 0,000 \\
Classe funcional & 0,021 \\
Níveis séricos de sódio & 0,119 \\
Níveis séricos de hemoglobina & 0,199
\end{tabular}


$\mathrm{Na}$ análise univariada os fatores que estiveram significativamente relacionados com sobrevida livre de eventos (óbito e internação) foram: intervenção, idade, etiologia chagásica, qualidade de vida basal, qualidade de vida pós, fração de ejeção de ventrículo esquerdo, classe funcional e hemoglogina. (Tabela 5)

Tabela 5 - Analise univariada dos fatores associados a eventos

\begin{tabular}{lc}
\hline Variáveis & Significância $(\mathrm{p})$ \\
\hline Intervenção & 0,000 \\
Gênero & 0,798 \\
Idade & 0,001 \\
Etiologia chagásica & 0,007 \\
Qualidade de vida basal & 0,074 \\
Qualidade de vida após randomização & 0,000 \\
Aderência & 0,164 \\
Fração de ejeção de ventrículo esquerdo & 0,011 \\
Classe funcional & 0,000 \\
Níveis séricos de sódio & 0,460 \\
Níveis séricos de hemoglobina & 0,047
\end{tabular}




\subsection{Analise Multivariada}

$\mathrm{Na}$ análise pelo modelo multivariado de risco proporcional de Cox, as variáveis que estiveram significativamente relacionadas à sobrevida foram: idade e fração de ejeção de ventrículo esquerdo. Quando excluímos a fração de ejeção da análise observamos que os fatores que estiveram relacionados com a sobrevida foram: gênero, idade, etiologia chagásica e qualidade de vida durante o seguimento. (Tabela 6)

Tabela 6 - Análise multivariada* dos fatores associados à sobrevida

\begin{tabular}{lc}
\hline Variáveis & Significância $(p)$ \\
\hline Gênero (sexo) & 0,009 \\
Idade & 0,007 \\
Etiologia chagásica & 0,042 \\
Qualidade de vida após randomização & 0,000
\end{tabular}

* Nesta análise não foi incluído a variável fração de ejeção do ventrículo esquerdo

$\mathrm{Na}$ análise pelo modelo multivariado de risco proporcional de Cox, as variáveis que estiveram significativamente relacionadas à sobrevida livre de eventos (óbito e internação) sem inclusão da fração de ejeção de ventrículo esquerdo foram: qualidade de vida durante o seguimento, etiologia chagásica e idade. (Tabela 7) 
Tabela 7- Análise multivariada - livre de eventos (óbito e internação) Variáveis Significância $(p)$

Qualidade de vida após randomização $\quad 0,000$

$\begin{array}{ll}\text { Etiologia chagásica } & 0,036\end{array}$

$\begin{array}{ll}\text { Idade } & 0,000\end{array}$ 


\subsection{Sobrevida}

O número de pacientes que faleceram durante o seguimento foi de $101(36,7 \%)$ no grupo intervenção e de $62(45,2 \%)$ no controle; foram submetidos a transplante cardíaco no grupo intervenção $18(4,5 \%)$, e no controle $8(5,8 \%)$ pacientes.

\subsubsection{Sobrevida}

A comparação das curvas de sobrevida entre grupo intervenção e controle não mostrou diferença significativa ( $p=n s)$.

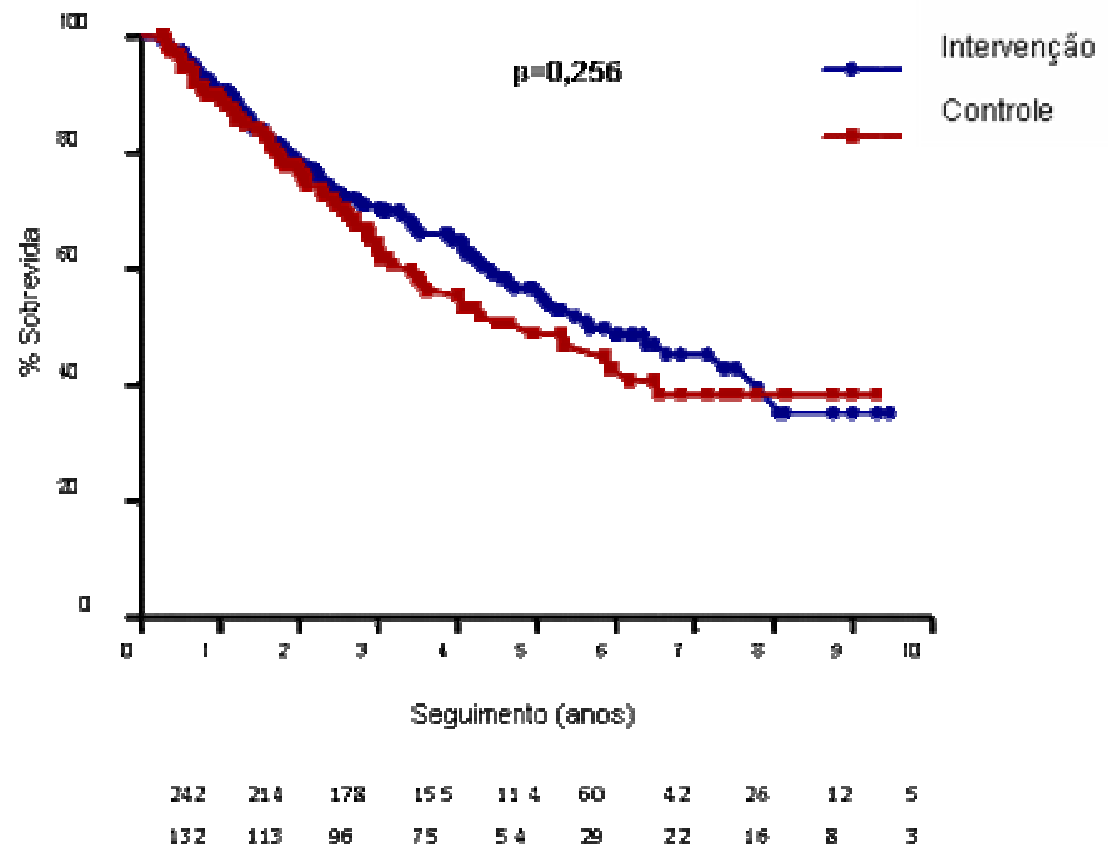

Figura 10. Curva de Sobrevida 


\subsubsection{Sobrevida Livre de Eventos}

Ao analisarmos a curva de sobrevida livre de eventos combinados (óbito e internação), observamos que ocorreu melhora significativa no grupo intervenção em relação ao grupo controle $(p=0,001)$.

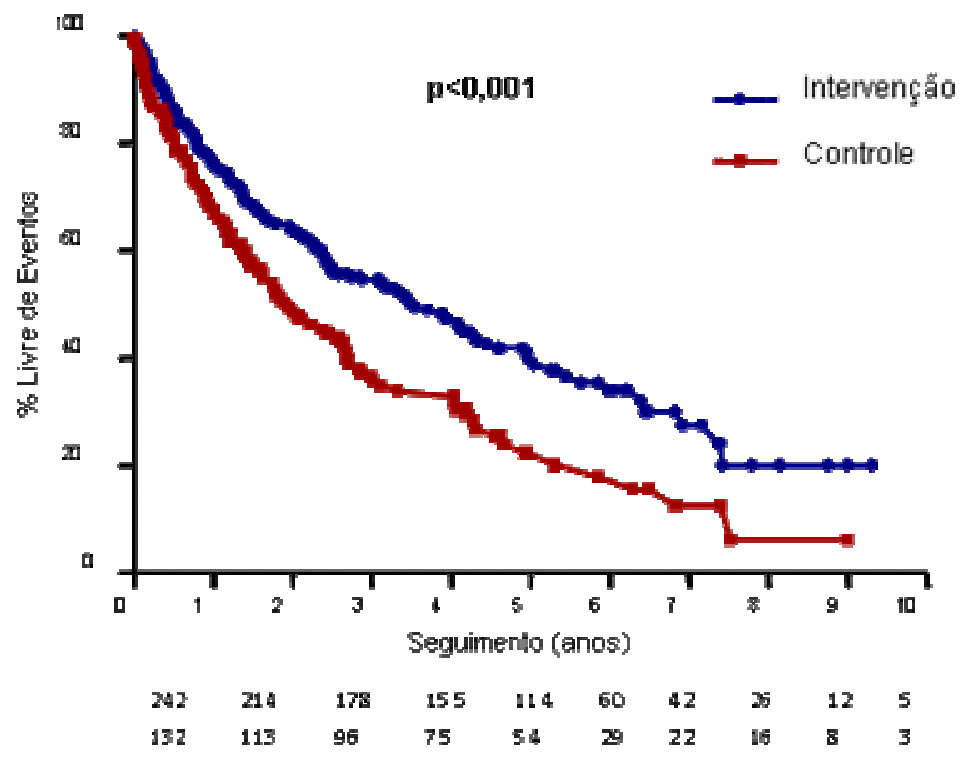

Figura 11. Curva de Sobrevida Livre de Eventos 


\subsubsection{Sobrevida e Qualidade de Vida Basal}

Ao analisarmos a curva de sobrevida conforme "quartis" da qualidade de vida basal não observamos diferença de sobrevida $(p=0,771)$.

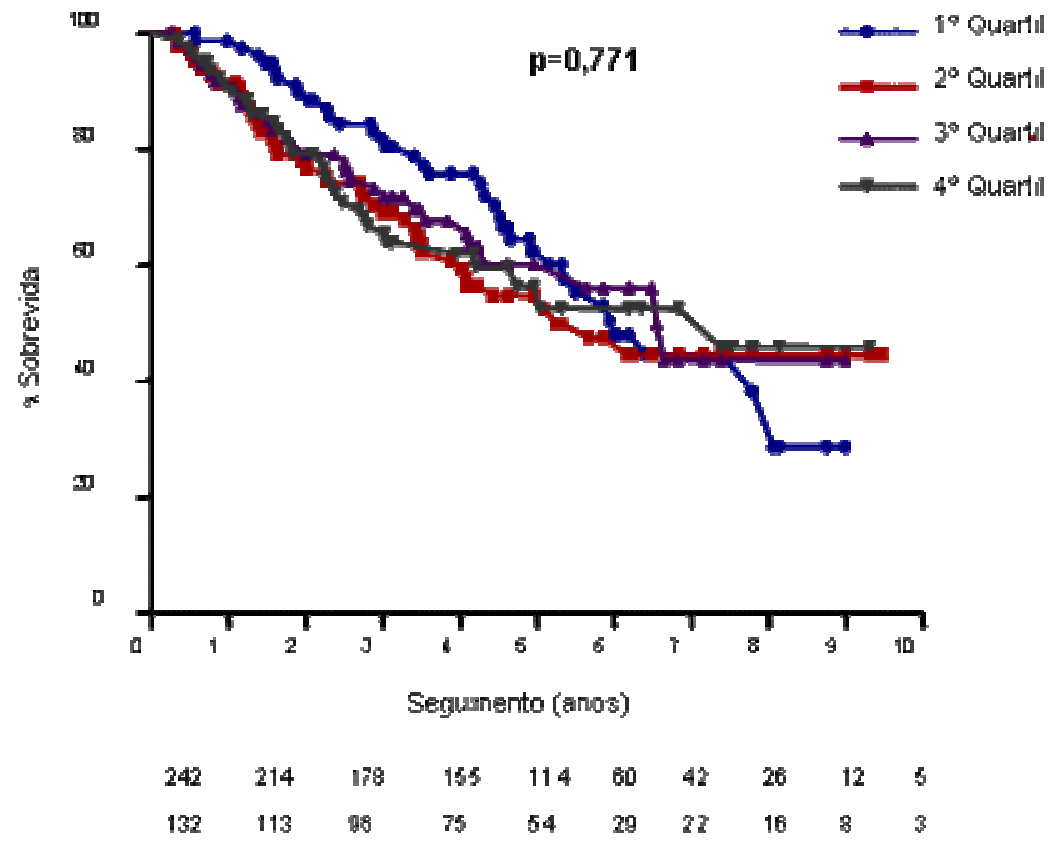

Figura 12. Curva de Sobrevida e Qualidade de vida basal 


\subsubsection{Sobrevida livre de eventos e Qualidade de Vida Basal}

Ao analisarmos a curva de sobrevida livre de eventos combinados (óbito e internação) conforme "quartis" da qualidade de vida basal não observamos diferença estatística. $(p=0,289)$.

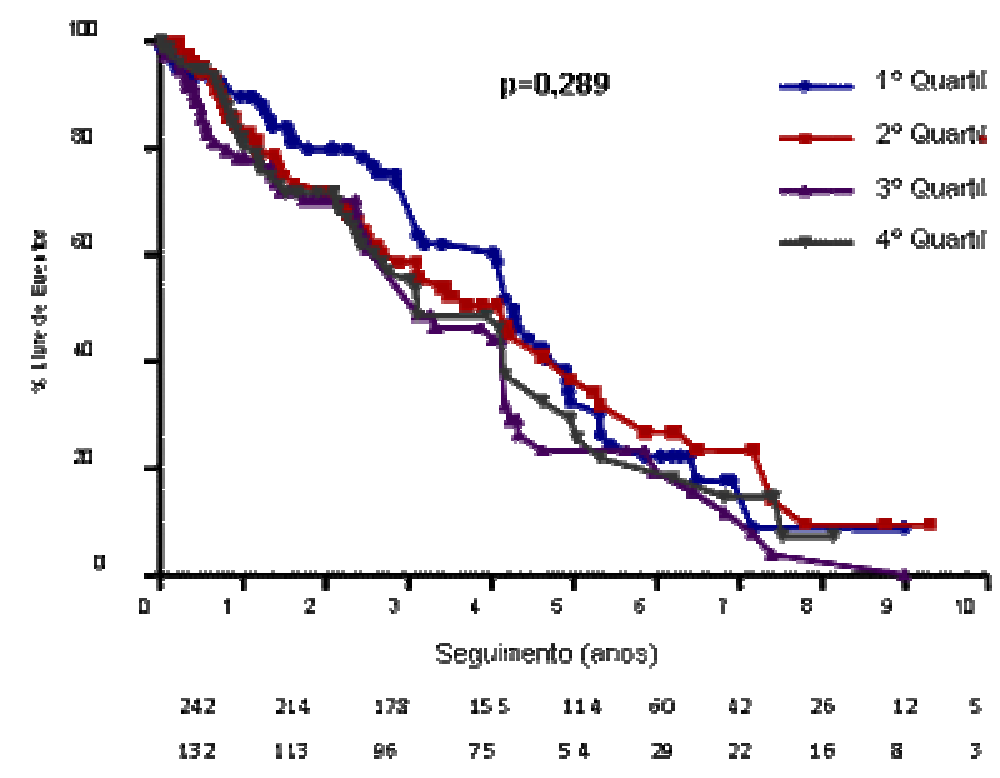

Figura 13. Sobrevida livre de eventos e qualidade de vida basal 


\subsubsection{Sobrevida e Qualidade de Vida no Seguimento}

Ao analisarmos a curva de sobrevida em "quartis" da qualidade de vida no seguimento observamos que ocorreu diferença estatisticamente significante $(p<0,001)$.

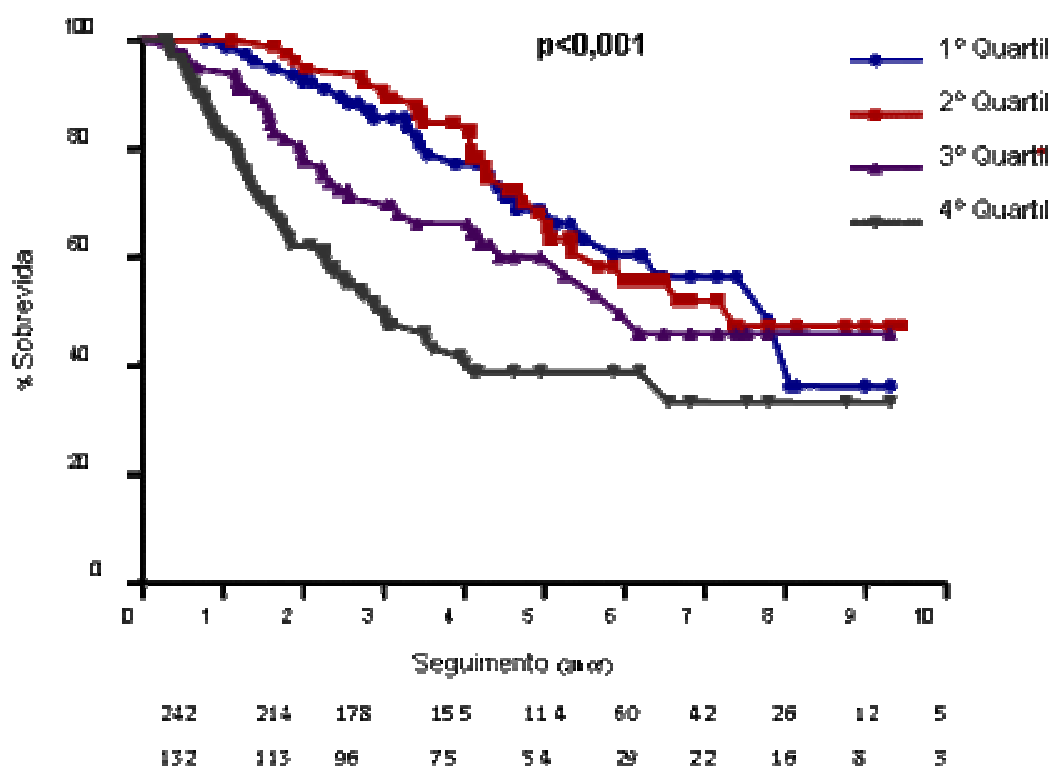

Figura 14. Curva de Sobrevida e Qualidade de Vida no seguimento 


\subsubsection{Sobrevida Livre de Eventos e Qualidade de Vida no Seguimento}

Ao analisarmos a curva livre de eventos combinados (óbito e internação) em "quartis" da qualidade de vida no seguimento observamos diferença estatisticamente significativa $(p=0,001)$.

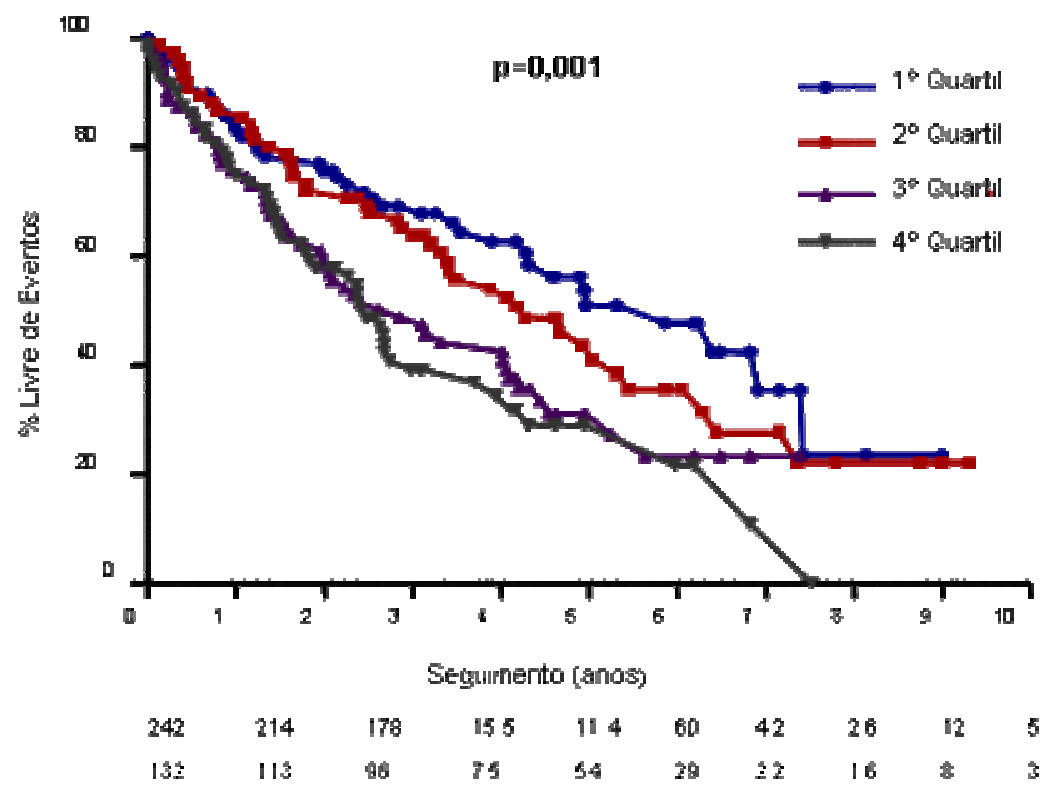

Figura 14. Curva de Sobrevida livre de eventos e Qualidade de Vida no seguimento 
5. Discussão 


\section{DISCUSSÃO}

Os resultados do presente estudo são pioneiros e demonstram que um programa contendo educação e monitorização repetitivos, desenvolvido para o nosso meio, é capaz de melhorar a QV em pacientes com IC no seguimento ambulatorial de longo prazo. Tem a característica se diferenciar por ser o único a realizar a avaliação seqüencial e de todos os componentes da QV, sob cuidados de equipe interdisciplinar e cardiologistas especializados em IC. A melhora da QV foi significativa tanto para o resultado global, como para cada um de seus componentes: dimensão física, dimensão emocional e outras questões que integram o MLWHFQ.

\section{Qualidade de Vida Global}

Os efeitos dos programas de educação e monitorização na QV de pacientes com IC tem sido polêmicos. Alguns estudos com programa de educação e monitorização demonstraram resultados benéficos coincidentes na avaliação final do seguimento, mas a não realizaram seqüencialmente de longo prazo, tiveram tempo médio de seguimento menor e incluíram pacientes após alta hospitalar. Estudo multicêntrico realizado com educação e monitorização contínua em pacientes com IC descreveu melhora da QV e suas dimensões. ${ }^{54}$ Entretanto, o tempo de seguimento médio foi menor (16 meses), foi limitado pelo número de eventos e a avaliação da QV não foi seqüencial e não incluiu todas as dimensões. De maneira contrastante com 
os nossos achados, outros autores não encontraram influência de programas de educação na QV de pacientes com IC. Investigadores que realizaram programa de educação em pacientes em acompanhamento ambulatorial com estabilidade clínica e seguimento de doze meses não encontraram melhora do resultado global na QV no final do estudo. ${ }^{55,56} \mathrm{Da}$ mesma forma, estudo incluindo pacientes após a alta hospitalar com programa de educação e seguimento de doze semanas; ${ }^{57}$ assim como estudo com educação e monitorização telefônica em pacientes com CF III e IV não demonstraram melhora da QV. ${ }^{58}$ Programa de intervenção por farmacêuticos comunitários também não demonstrou melhora da $Q V ;$ o estudo compreendeu uma visita após duas semanas da alta hospitalar com orientação, e a outra após seis a oito semanas com reforço de educação, sendo que a avaliação da qualidade de vida foi realizada no final do estudo. 59 Além de serem diferentes na metodologia, na estrutura do programa, na situação sócio-econômica e cultural, outro fator que pode justificar tais diferenças foi a inclusão no nosso estudo de todas as etiologias, entre elas a etiologia chagásica predominante na América Latina, assim como de pacientes mais jovens.

\section{Dimensões da Qualidade de Vida}

Em nosso estudo todos os componentes da qualidade de vida apresentam-se comprometidos pela doença, tanto no aspecto físico, emocional e global, mas muitos estudos que realizaram a avaliação da qualidade vida não incluíram sequencialmente de longo prazo a avaliação de 
todos os componentes que incluem além das dimensões emocionais e físicas, as outras questões que formam o resultado global. ${ }^{60,61}$

Os componentes da dimensão emocional apresentaram melhora inicial já aos seis meses de seguimento, embora a melhora tenha sido estatisticamente significativa a partir dos dezoito meses de seguimento. Não existem publicações a respeito do tempo de melhora dos componentes da dimensão emocional para comparação. Nosso resultado provavelmente pode estar relacionado às limitações ocasionadas por fatores extrínsecos que estão interligadados diretamente à doença, como aspectos psicológicos e sócio-econômicos que podem influenciar e contribuir para as expectativas do paciente e no resultado do tratamento. Pacientes com insuficiência cardíaca apresentam alta prevalência de depressão, ansiedade, e distúrbios psicológicos que podem ter ser sido alvo deste programa de educação e monitorização repetitivos. ${ }^{62}$ Recentemente, foi demonstrado que a depressão pode ter valor independente na determinação de mortalidade e hospitalização no seguimento de pacientes com IC.$^{63}$

Nos componentes da dimensão física foram observados melhora estatisticamente significante somente aos vinte e quatro meses de seguimento, portanto mais tardia do que a melhora dos componentes emocionais. Não existem resultados publicados a esse respeito na literatura. Este achado provavelmente está relacionado às limitações de melhora na IC em uma fase inicial, da congestão pulmonar, do edema e outros mecanismos levando à dispnéia e baixa perfusão. Em pacientes já acompanhados por especialistas em insuficiência cardíaca, sob seguimento 
ambulatorial usual onde já existe orientação para aspectos associados à congestão, este programa pode ser mais lento na obtenção do efeito benéfico. Entretanto, nossos resultados devem estimular o aperfeiçoamento de programas de educação e monitorização para que sejam mais efetivos precocemente neste aspecto.

Nas outras questões que somadas à dimensão física e emocional compõe o resultado global, não encontramos estudos que tenham descrito a sua resposta em programas de educação e monitorização na IC.

\section{Qualidade de Vida e Sobrevida}

Em nosso estudo a qualidade de vida basal dos pacientes não esteve relacionada à sobrevida ou a chance de eventos combinados. Nosso resultado diverge de resultados de outros estudos que demonstraram relação entre qualidade de vida e sobrevida. ${ }^{64,65,66}$ Entretanto, houve relação entre a qualidade de vida após a randomização e a sobrevida em nossos resultados.

Entre os possíveis fatores que podem explicar esta divergência numa fase prévia a randomização em relação a outros estudos representando uma população usual estão a gravidade maior de pacientes incluídos em outros estudos, diferentes etiologias incluídas, diferenças sociais, de escolaridade, culturais, e tempo de seguimento menor em outros estudos. No entanto, a relação observada após a randomização em nossos resultados demonstra que esse programa de educação e monitorização 
influencia componentes associados à sobrevida e qualidade de vida. Se a qualidade de vida é um marcador ou tem papel direto causando óbito ou sobrevida não é conhecido. Em nosso estudo nos modelos estatísticos de análise multivariada em que a fração de ejeção não foi incluída, a qualidade de vida obteve importância independente, ou seja, em situações clínicas em que não fração de ejeção a qualidade de vida é um marcador do prognóstico do paciente.

Em estudo recente já na era dos beta-bloqueadores, que incluiu avaliação da qualidade de vida mensurada de maneira seqüencial e análise do prognóstico, observou-se relação da qualidade de vida com desfecho combinado de mortalidade e hospitalização. ${ }^{67}$ Entretanto, neste estudo em que a qualidade de vida foi mensurada em 1050 pacientes afro-americanos aos 3 e 6 meses de seguimento não houve relação com mortalidade. Saliente-se que este estudo focou-se em grupo étnico específico, e com tempo de seguimento inferior ao do nosso estudo.

\section{Qualidade de Vida e Gênero}

Em nosso estudo, a avaliação da qualidade de vida entre os gêneros demonstrou melhora significativa durante o seguimento no grupo intervenção em ambos os sexos, entretanto no gênero masculino a melhora ocorreu tardiamente em relação ao gênero feminino. Em contraste com os nossos resultados, o estudo mostrou que mulheres com insuficiência cardíaca apresentaram menor qualidade de vida durante o seguimento de 
um ano, após programas de educação e monitorização. ${ }^{68} \mathrm{~A}$ maior prevalência de depressão, ansiedade, e medo nos pacientes do sexo feminino em comparação com o sexo masculino poderia explicar esta melhora mais precoce. ${ }^{69}$

\subsection{IMPLICAÇÃOES CLÍNICAS}

A demonstração da melhora dos componentes da QV em pacientes com IC submetidos a um programa repetitivo de educação e monitorização pode ter um papel fundamental no sucesso da terapêtica da insuficiência cardíaca. A não inclusão de pacientes após alta hospitalar, portanto em seguimento ambulatorial, introduz uma nova indicação adequada para um programa desenvolvido em nosso meio.

A demonstração de que as diferentes dimensões podem melhorar de maneira diferente no tempo, podem estimular precocemente a identificação e o desenvolvimento de novas estratégias e intervenções nos programas de educação e monitorização na insuficiência cardíaca.

A análise da QV basal na nossa casuística não demonstrou ter valor prognóstico, entretanto, a relação entre mortalidade e qualidade de vida após a randomização sugere que, mesmo na presença da dúvida, se a qualidade de vida é só um marcador ou tem valor causal, deve ser alvo importante do tratamento para tentar reduzir mortalidade ou eventos. 


\subsection{LIMITAÇÕES DO ESTUDO}

A QV caracteriza-se por ser subjetiva e multidimensional, podendo assim, apresentar limitações em sua avaliação e análise provenientes deste tipo de estudo.

Limitações do estudo podem estar relacionadas ao fato do projeto ser aberto, e não ser cego quanto à alocação da intervenção. O estudo não cego pode desencadear certo grau de co-intervenção pela equipe interdisciplinar, podendo influenciar na compensação dos pacientes que não participam do grupo intervenção. Contudo, os pacientes do grupo controle poderiam ter contato com os pacientes do grupo intervenção e obter informações sobre as intervenções realizadas. 
6. Conclusão 


\section{CONCLUSÔES}

Este estudo demonstra que um programa repetitivo seqüencial de educação e monitorização, desenvolvido para o nosso meio e implementado por equipe interdisciplinar e de longo prazo, pode ser aplicado para melhoria de todos os componentes da qualidade de vida de pacientes com IC.

A demonstração de que este programa de educação e monitorização pode influenciar de maneira diferente pacientes do sexo masculino e feminino, deve estimular o desenvolvimento de programas individualizados a serem testados para cada sexo.

Nossa análise, demonstrando que os componentes da qualidade de vida podem responder de forma heterogênea às intervenções, contribui para o desenvolvimento de intervenções dirigidas precocemente destes aspectos que têm resposta mais lenta.

A demonstração de que a qualidade de vida após a randomização tem valor prognóstico durante o seguimento, mesmo na dúvida se há causaefeito, deve estimular cada vez mais que a melhoria da qualidade de vida seja um objetivo maior no tratamento da insuficiência cardíaca. 
7. Anexos 


\section{ANEXO 1}

MINNESOTA LIVING WITH HEART FAILURE QUESTIONNAIRE

Nome: RG:

Durante o Último Mês seu Problema Cardíaco o Impediu de Viver como Você Queria Por Quê?

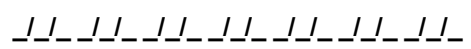

Pré $6 \mathrm{~m} \quad 12 \mathrm{~m} \quad 18 \mathrm{~m} \quad 24 \mathrm{~m} \quad 36 \mathrm{~m} \quad 48 \mathrm{~m}$

1. Causou inchaço em seus tornozelos e pernas

()()()()()()()

2. Obrigando você a sentar ou deitar para descansar durante o dia ( ) ( ) ( ) ( ) ( ) ( ) ( )

3. Tornando sua caminhada e subida de escadas difíceis

( ) ( ) ( ) ( ) ( ) ( ) ( )

4. Tornando seu trabalho doméstico difícil

( ) ( ) ( ) ( ) ( ) ( ) ( )

5. Tornando suas saídas de casa difíceis

( ) ( ) ( ) ( ) ( ) ( ) ( )

6. Tornando difícil dormir bem a noite

( ) ( ) ( ) ( ) ( ) ( ) ( )

7. Tornando seus relacionamentos ou atividades com familiares

$($ ) ( ) ( ) ( ) ( ) ( ) ( )

e amigos difíceis

8. Tornando seu trabalho para ganhar a vida difícil

( ) ( ) ( ) ( ) ( ) ( ) ( )

9. Tornando seus passatempos, esportes e diversão difíceis

( ) ( ) ( ) ( ) ( ) ( ) ( )

10. Tornando sua atividade sexual difícil

( ) ( ) ( ) ( ) ( ) ( ) ( )

11. Fazendo você comer menos as comidas que você gosta

( ) ( ) ( ) ( ) ( ) ( ) ( )

12. Causando falta de ar

13. Deixando você cansado, fatigado ou com pouca energia

( ) ( ) ( ) ( ) ( ) ( ) ( )

14. Obrigando você a ficar hospitalizado

( ) ( ) ( ) ( ) ( ) ( ) ( )

15. Fazendo você gastar dinheiro com cuidados médicos

( ) ( ) ( ) ( ) ( ) ( ) ( )

16. Causando a você efeitos colaterais das medicações

( ) ( ) ( ) ( ) ( ) ( ) ( )

17. Fazendo você sentir-se um peso para familiares e amigos

( ) ( ) ( ) ( ) ( ) ( ) ( )

18. Fazendo você sentir uma falta de autocontrole na sua vida

( ) ( ) ( ) ( ) ( ) ( ) ( )

19. Fazendo você se preocupar

$($ ) ( ) ( ) ( ) ( ) ( ) ( )

20. Tornando difícil você concentrar-se ou lembrar-se das coisas

$($ ) ( ) ( ) ( ) ( ) ( ) ( )

21. Fazendo você sentir-se deprimido

$($ ) ( ) ( ) ( ) ( ) ( ) ( )

$($ ) ( ) ( ) ( ) ( ) ( ) ( ) 
GABARITO

MINNESOTA LIVING WITH HEART FAILURE QUESTIONNAIRE

NÃO

0

1

2

4

5 


\section{Apêndice}


8.1 APENDICE 1 - Fotos dos pacientes da clínica de insuficiência em reunião com a equipe interdisciplinar

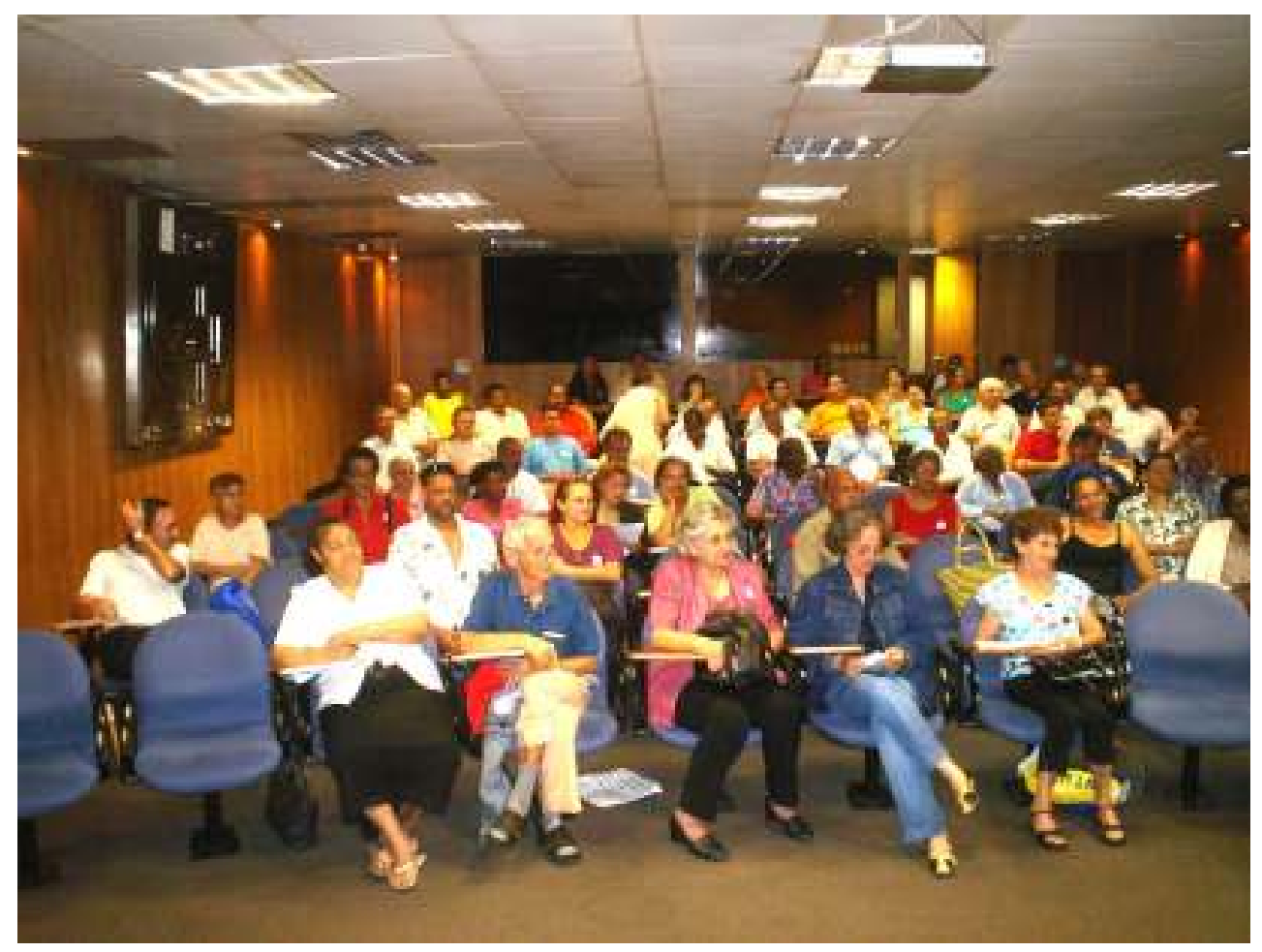




\section{Referencias Bibliográficas}




\section{REFERENCIAS BIBLIOGRÁFICAS}

1. Bocchi EA, Marcondes FBG, Ayub SFM, Rohde LE, Oliveira WA, Almeida DR, e cols. Sociedade Brasileira de Cardiologia. III Diretriz Brasileira de Insuficiência Cardíaca Crônica. Arq Bras Cardiol 2009;92(6 supl. 1):1-71.

2. Bocchi EA, Mocelin A. Importância da Terapêutica Otimizada no tratamento da Insuficiência Cardíaca Sístólica. Rev Soc Cardiol Estado de São Paulo $\quad$ 2000;10(1):56-61.

3. Swedberg K, Cleland J, Dargie H, Drexler H, Follath F, Komajda M, et al. Task Force for the Diagnosis and Treatment of Chronic Heart Failure of the European Society of Cardiology. Guidelines for the diagnosis and treatment of chronic heart failure: executive summary (update 2005): The Task Force for the Diagnosis and Treatment of Chronic Heart Failure of the European Society of Cardiology. Eur Heart J 2005;26:1115-40.

4. Aaronson KD, Schwartz JS, Chen TM, Wong KL, Goin JE, Mancini DM. Development and prospective validation of a clinical index to predict survival in ambulatory patients referred for cardiac transplant evaluation. Circulation 1997;95:266.

5. Butler J, Khadim G, Paul KM, Davis SF, Kronenberg MW, Chomsky DB, Pierson RN , Wilson JR. Selection of patients for heart transplantation in 
the current era of heart failure therapy. J Am Coll Cardiol 2004;43:78793.

6. Wilson JR, Rayos G, Yeoh TK, Gothard P, Bak K. Dissociation between exertional symptoms and circulatory function impatiens with heart failure. Circulation 1995;92:47-53.

7. Cowie MR - The epidemiology of heart failure - An epidemic in progress. In: Coats A, Cleland JGF (ed). Controversies in the management of heart failure. Churchill Livingstone 1997; p.11-23.

8. Moser DK \& Worster PL. Effect of psychosocial factors on physiologic outcomes in patients with heart failure. Journal of Cardiovascular Nursing 2000;14:106-115.

9. Jessup M, Brozena S. Heart Failure. Review article. N Engl J Med 2003;348:2007-18.

10. DATASUS. Disponível em http// www.datasus.org.br.

11. Albanesi Filho, F.M. - O que vem ocorrendo com a insuficiência cardíaca no Brasil? Arq Bras Cardiol 2005;85:155-6. 
12. Sneed, NV, Paul S, Michel Y, Vanabakel A, Hendrix F. Evaluation of quality of live Measuremente tools in patients with chronic heart failure. Heart Lung 2001, 30:332-40

13. Butler J, Khadim G, Paul KM, Davis SF, Kronenberg M W, Don B. Chomsky DB, Pierson RN, Wilson JR. Selection of patients for heart transplantation in the current era of heart failure therapy. J Am Coll Cardiol 2004;43:787-93.

14. Hanumanthu S, Butler J, Chomsky D, Davis S, Wilson JR. Effect of a heart failure program on hospitalization frequency and exercise tolerance. Circulation 1997; 96:2842-8.

15. The SOLVD Investigators. Effect of enalapril on mortality and development of heart failure in asymptomatic patients with reduced left ventricular ejection fraction. N Engl J Med 1992;327:685 - 91.

16. The SOLVD Investigators. Effect of enalapril on survival in patients with reduced left ventricular ejection fraction and congestive heart failure. $N$ Engl J Med 1991;327:293 - 302. 
17. Packer M, Bristow MR, Cohn JN, Colucci WS, Fowler MB, Gilbert EM, Shusterman $\mathrm{NH}$. Effect of carvedilol on morbidity and mortality in chronic heart failure. N Engl J Med 1996; 334:1349-55.

18. The CIBIS-II Investigators and Committees. The cardiac insufficiency bisoprolol study II (CIBIS-II): a randomized trial. Lancet 1999; 353:9-13.

19. Merit-HF Study Group. Effect of metroprolol CR/XL in chronic heart failure: Metroprolol $\mathrm{Cr} / \mathrm{XL}$ randomized intervention trial in congestive heart failure (MERIT-HF). Lancet 1999;353:2001-7.

20. Packer M, Coats AJ, Fowler, MB, Katus HA, Krum H, Mohacsi P, Rouleau JL, Tendera M, Castaigne A, Roecker EB, Schultz MK \& DeMets DL. Effect of carvedilol on survival in severe chronic heart failure. N Engl J Med 2001;347:1397-402.

21. Levy D, Kenchaiah S, Larson MG, Benjamin EJ, Kupka MJ, Ho KK, Murabito JM, Vasan R. Long-term trends in the incidence of and survival with heart failure. N Engl J Med 2002;347:1397-402. 
22 Fonarow GC, Stevenson LW, Walden JA, Livingston AN, Steimle AE, Hamilton MA, Moriguchi J, Tillisch JH, Woo MA. Impact of a comprehensive heart failure management program on hospital readmission and functional status of patients with advanced heart failure. J Am Coll Cardiol 1997;30:518-26.

23. Roccaforte R, Demers C, Baldassarre F, Teo KK, Yusuf S. Effectiveness of comprehensive disease management programmes in improving clinical outcomes in heart failure patients. A meta-analysis.[see comment][erratum appears in Eur J Heart Fail. 2006 Mar;8(2):223-4]. European Journal of Heart Failure 2005 December;7(7):1133-44.

24. Rich MW, Beckham V, Wittenberg C, Leven CL, Freedland KE, Carney RM. A multidisciplinary intervention to prevent the readmission of elderly patients with congestive heart failure. [see comment]. New England Journal of Medicine 1995 November 2;333(18):1190-5.

25. Néri AL. Qualidade de Vida na Velhice e Atendimento Domiciliário. In: Atendimento Domiciliar: Um enfoque Gerontológico. São Paulo: Atheneu 2000; 33-47. 
26. Stromberg A. Educating nurses and patients to manage heart failure. Eur J Cardiovasc Nurs 2002;1:33-40.

27. Riegel B, Carlson B, Kopp Z, LePetri B, Glaser D, Unger A. Effect of a standardized nurse case-management telephone intervention on resource use in patients with chronic heart failure. [see comment]. Archives of Internal Medicine 2002 March 25;162(6):705-12.

28. Gonseth J, Guallar-Castillon P, Banegas JR, Rodriguez-Artalejo F. The effectiveness of disease management programs in reducing hospital readmission in older patients with heart failure: a systematic review and meta-analysis of published reports. [see comment]. [Review] [113 refs]. Eur Heart J 2004 September;25(18):1570-95.

29. Giliarevskii SR OVKLBNBOSE. [Effect of therapeutic education of patients with chronic heart failure on quality of life and requirement of rehospitalizations. Results of 12-months randomized study]. Kardiologiia 2002;(5):56-61.

30. Bocchi EA. Heart failure clinics: the Brazilian Experience. Rev Port Cardiol 2004;23:suppl III:47-55. 
31. Bocchi EA. Clínica de Insuficiência Cardíaca. In: Barreto, A. C. P.; Bocchi, E. A. (Org.). Insuficiência Cardíaca. São Paulo, 2003 v. 1, p.269277

32. Uretsky BF,Pina I,Quigg RJ,Brill JV,Maclnerney EJ,Mintzer R,Armstrong PW. Beyond drug therapy: Nonpharmacologic care of the patient with advanced heart failure. Am Heart J 1998;135:S264-84.

33. Erhardt LR, Cline CM. Organization of the care of patients with heart failure. Lancet 1998;352(supp II):15-8.

34. Cruz FD, Nakassato M, Issa, VS. Tratamento não medicamentoso da insuficiência cardíaca - Como melhorar a qualidade de vida. In: Barreto, A. C. P.; Bocchi, E. A. (Org.). Insuficiência Cardíaca. São Paulo, 2003, v. 1, p.151-159.

35. Bocchi EA, Cruz FD, Guimarães GV, Moreira LF, Issa VS, Ferreira AS, Emilio GC, Chizzola PR, Brandão S, Bacal F. Long-Term Prospective, Randomized, Controlled Study Using Repetitive Education at Six-Month Intervals and Monitoring for Adherence in Heart Failure Outpatients REMADHE. Circulation 2008;1:115-124. 
36. Beck P, Jensen MN, Obel EB, Ulrich LG, Eiken P, Nielsen SPors. Quality of life measurements for patients taking which drugs? The clinical PCASEE perspective. PharmacoEconomics. 1995;7(2):141-151.

37. Ferrans CE e POWERS M.J. Psychometric Assessment of the Quality of Life Index. Research in Nursing \& Health 1992;15:29-38.

38. Padilla GV, Kagawa- Singer M. Quality of Life and Culture. In: King CR, Hinds PS. Quality of Life from Nursing and Patient Perspectives: theory, research, practice. London: Jones and Bartlett 1998;5:74-92.

39. Goodinson SM, Singleton JBA. A Quality of life: a Critical Review of Current Concepts, Measures and Their clinical Implications. J Nursing Study 1989;26(4):327-341.

40. Gill TM, Feinstein AR. A Critical Appraisal of the Quality of Quality-of-life Measurements. JAMA 1994;272: 619-626.

41. Rector TS, Cohn JN. Assessment of patients outcome with the Minnesota Living with Heart Failure Questionnaire: Reliability and Validity during a randomized, double-blind, placebo-controlled trial of pimobedan. Am Heart J 1992;124:1017-25. 
42. Rector TS, Kubo SH, Cohn JN. Patients self-assessment of their congestive heart failure: II. Content, reliability and validity of a new measure - the Minnesota Living with Heart Failure questionnaire. Heart Failure. 1987; 3:198-209.

43. Lewis EF, Johnson PA, Johnson W, Collins C, Griffin L, Stevenson LW. Quality of life. Preferences for quality of life survival expressed by patients with heart failure. Heart Lung Transplantation J 2001; 20(9):1016-24.

44. Rector TS, Kubo SH, Cohn JN. Validity of the Minnesota Living with Heart Failure Questionnaire as a measure of therapeutic response to enalapril or placebo. Am J Cardiol. 1993;71:1106-7.

45. Rector TS, Kubo SH, Cohn JN. Patients self-assessment of their congestive heart failure: II. Content, reliability and validity of a new measure - the Minnesota Living with Heart Failure questionnaire. Heart Failure 1987; 3:198-209.

46. Carrara D. Avaliação prospectiva de qualidade de vida em pacientes com miocardiopatia dilatada submetidos à ventriculectomia parcial esquerda [dissertação de mestrado]. São Paulo: Faculdade de Medicina da Universidade de São Paulo; 2001. 
47. Carvalho VO, Guimarães GV, Carrara D, Bacal F, Bocchi E. Validação da versão em português do Minnesota Living with Heart Failure Questionaire. Arq. Bras. Cardiol 2009; 93(1): 36-41

48. Johansson P, Agnebrink M., Dahlstro M, \& Brostrom A. Measurement of health-related quality of life in chronic heart failure, from a nursing perspective - a review of the literature. European Journal of Cardiovascular Nursing 2004; 3(1),7-20.

49. Jaarsma T, Halfens R, Abu-Saad HH, Dracup K, Stappers J, Ree V. Qality life in older patients with systolic and diastolic heart failure. The European Journal of Heart Failure 1999;151-60.

50. Ho KK; Anderson KM; Kannel WB; Grossman W; Levy. Survival after the onset of congestive heart failure in Framingham heart study subjects. Circulation. 1993; 88:107-15.

51. Jaagosild P; Dawson NV; Thomas C; Wenger NS; Tsevat J; Knaus WA; Califf RM; Goldman L; Vidaillet H; Connors AF. Outcomes of acute exacerbation of severe congestive heart failure: quality of life, resource use, and survival. Arch Intern Med 1998;158:1081-89.

52. Vieira A, Hossne S. Diretrizes e Normas da Resolução n. 196/1966. Conselho Nacional de Saúde - Ministério da Saúde, 1998. 
53. Charles WT, Robertson AD, Zolty Ronald, Shakar SF, Lindenfeld J, Wolfel EE, Bristow MR, Lowes BD. Quality of life and prognosis in heart failure: Results of the beta-blocker Evaluation of Survival Trial (BEST). $J$ Cardiac Falilure 2007;13:732-7.

54. Granceli HO. Disease management programs in heart failure. Findings of the DIAL study. Rev. Esp. Cardiol 2007;60 3):15-22.

55. DeWalt DA, Malone RM, Bryant ME, Kosnar MC, Corr KE, Rothman RL, Sueta CA, Pignone MP. A heart failure self-management program for patients of all literacy levels: a randomized, controlled trial [ISRCTN11535170]. BMC Health Serv Res. 2006;6:30.

56. Harrison MB, Browne GB, Roberts J, Tugwell P, Graham ID. Quality of life individuals with heart failure: a randomized trial of the effectiveness of two models of hospital-to-home transition. Med Care 2002;40(4):271281.

57. Goldberg L, Piette J, Walsh M. Randomized trial of a daily electronic home monitoring system in patients with advanced heart failure: The Weight Monitoring in Heart failure (WHARF) trial. Am Heart $J$ 2003;146:705-712. 
58. McAlister FA, Lawson FM, Teo KK, Armstrong PW. A systematic review of randomized trials of disease management programs in heart failure. $A$ J Med 2001;110:378-84.

59. Holland R, Battersby J, Harvey I, Lenagham E, smith J, Hay L. Systematic review of multidisciplinary intervention heart failure. Am Heart J 2005;91: 899-906.

60. Gwarry-Sridhar FH, Flinoft V, Lee DS, Lee, Guyatt GH. A systematic review a meta-analysis of studies comparing readmission rates and mortality rates in patients with heart failure: a systematic review and meta-analysis of published reports. Eur Heart J 2004;64:2315-20.

61. Morgan K, McGee H, Shelley E. Quality of life assessment in heart failure interventions: a 10-year (1996-2005) review. European Journal of cardiovascular Prevention \& Rehabilitation 2007;14(5):589-607.

62. Heo S, Moser DK, Widener. Gender differences in the effects of physical and emotional symptoms on health-related quality of life in patients with heart failure. Eur J Cardiovasc Nurs. 2007;6:146-52. 
63. Lesman-Leegte I, van Veldhuisen DJ, Hillege HL, Moser D, Sanderman $\mathrm{R}$, Jaarsma T.Depressive symptoms and outcomes in patients with heart failure: data from the COACH study. Eur J Heart Fail. 2009 ;11:1202-7.

64. Konstam V, Salem D, Pouleur H, Kostis J, Gorkin L, Shumaker S, Mottard I, Woods P, Konstam MA, Yusuf S. Baseline quality of life as predictor of mortality and hospitalization in 5,025 patients with congestive heart failure. SOLVD Investigations. Studies of left ventricular Dysfunction investigators. Am J Cardiol 1996, Oct 15;78(8):890-5.

65. Heidenreich PA, Spertus JA, Jones PG, Weintraub WS, Rumsfeld JS, Rathore SS, Peterson ED, Masoudi FA, Krumholz HM, Havranek EP, Conard MW, Williams RE. Cardiovascular Outcomes Research Consortium. Health status identifies heart failure outpatients at risk for hospitalization or death. J Am Coll Cardiol 2006;47:752-6.

66. Parissis JT, Nikolaou M, Farmakis D, Parakevaidis IA, Bistola V, Venetsanou K, Katsaras D, Filippatos G, Kremastinos DT. Selfassessment of health status is associated with inflammatory activation and predicts long-term outcomes in chronic heart failure. Eur J Heart Fail 2009; Feb 11(2):163-9. 
67. Carson P, Tam SW, Ghali Jk, Archambault WT, Taylor A, Cohn JN, Braman VM, Worcel M, Amand IS. Relationship of quality of life scores with baseline characteristics and outcomes in African-Americam heart falilure trial. J Card Fail 2009; Dec 15(10):835-42.

68. Marshall H. Chin MD, Goldman L. Gender differences in 1-year survival and quality of life among patients admitted with congestive heart failure. Medical Care 1998;36:1033-46.

69. Lesman-Leegte I, Jaarsma T, Coyne JC, Hillege HL, Van Veldhuisen DJ, Sanderman R. Quality of life and depressive symptoms in the elderly: a comparison between patients with heart failure and age- and gendermatched community controls.J Card Fail 2009 ;15:17-23. 